\title{
Strain rate and temperature effects on the selection of primary and secondary slip and twinning systems in $\mathrm{HCP} \mathrm{Zr}$
}

\author{
Marko Knezevic, ${ }^{\mathrm{a}, *}$, Milovan Zecevic ${ }^{\mathrm{a}}$, Irene J. Beyerlein ${ }^{\mathrm{b}}$, \\ John F. Bingert ${ }^{\mathrm{c}}$, Rodney J. McCabe \\ ${ }^{a}$ Department of Mechanical Engineering, University of New Hampshire, Durham, NH 03824, USA \\ ${ }^{\mathrm{b}}$ Theoretical Division, Los Alamos National Laboratory, Los Alamos, NM 87545, USA \\ ${ }^{c}$ Materials Science and Technology Division, Los Alamos National Laboratory, Los Alamos, NM 87545, USA
}

\begin{abstract}
We investigate the temperature and rate dependence of slip, twinning, and secondary twinning in high-purity hexagonal close packed $\alpha-Z r$ over a wide range of temperatures and strain rates (from $76 \mathrm{~K}$ to $673 \mathrm{~K}$ and $0.001 \mathrm{~s}^{-1}$ to $4500 \mathrm{~s}^{-1}$ ). To reliably identify the dominant deformation mechanisms for each condition, we employ electron-backscattered diffraction (EBSD), dislocation theory, multi-scale polycrystal constitutive modeling, and a thermally activated dislocation density evolution based hardening law. We demonstrate with direct comparison with measurement that the constitutive model, with a single set of intrinsic material parameters, can predict the underlying texture evolution, primary and secondary slip and twin activity, and twin volume fraction associated with the different loading orientations and applied temperatures and strain rates. We find that the $\{10 \overline{1} 2\}\langle 10 \overline{1} \overline{1}\rangle$ twin is the preferred tension twin, either as a primary or secondary twin depending on the sample orientation, over the broad temperature and strain rate range tested. In contrast, we show that the preferred contraction twin, whether $\{11 \overline{2} 2\}\langle 11 \overline{2} \overline{3}\rangle$ or $\{10 \overline{1} 1\}\langle 10 \overline{1} \overline{2}\rangle$, is sensitive to temperature but insensitive to strain rate and whether it is a primary or secondary twin. Based on the concomitant changes in the dominant slip mode predicted by the model and revealed by the texture development, we rationalize that the temperature-induced transition in contraction twinning is due to the increased predominance of basal <a> slip at high temperatures $(>673 \mathrm{~K})$. Last, our analysis implies that all twin modes studied are rate insensitive and so the strong influence of strain rate and temperature on twinning is due to the rate-sensitivity of slip.
\end{abstract}

Keywords: Hexagonal close packed; Zirconium; Twinning; Dislocations; Crystal plasticity

\footnotetext{
* Corresponding author at: University of New Hampshire, Department of Mechanical Engineering, 33 Academic Way, Kingsbury Hall, W119, Durham, New Hampshire 03824, United States. Tel.: 603 862 5179; fax: 603862 1865; email: marko.knezevic@unh.edu
} 


\subsection{Introduction}

In hexagonal close packed (HCP) metals, deformation twinning is an important deformation mechanism that governs texture evolution, hardening, and failure for many metals and their alloys and under various loading conditions [1,2]. Within a given grain in a polycrystalline metal, twinning typically acts in combination with plastic slip to accommodate deformation. Twins abruptly reorient the crystal lattice of the parent grain. The reoriented twin domain introduces a twin boundary, which interacts with subsequent slip, and, within the reoriented twin domains, secondary slip and/or secondary twinning can occur with continued straining. Taken together, these two basic effects of twinning can significantly contribute to material strain hardening in at least three ways: (i) the hardening/softening due to crystal reorientation, from geometrically softer to harder orientations and vice versa [3-6], (ii) the Hall-Petch-like hardening due to grain subdivision [4, 7-9], and (iii) the Basinski-type hardening mechanism due to transmutations of dislocations from glissile to sessile [10]. Thus, understanding and predicting the mechanical behavior and microstructure evolution of HCP metals require appropriate models for twinning and de-twinning within polycrystal constitutive models.

To this end, four approaches currently exist in the literature for treatment of reoriented deformation twin domains within polycrystal plasticity codes: (i) the predominant twin reorientation (PTR) method [11, 12], (ii) the volume fraction transfer (VFT) scheme [12], (iii) the total Lagrangian approach [13, 14], and (iv) the composite-grain method [7]. Mean-field crystal plasticity models, such as Taylor-type [15-22] and self-consistent [23-25] schemes, are usually used as platforms in which these schemes are incorporated. Together, they provide a way to model the effects of deformation twinning on constitutive response [23, 26-30]. They typically

allow for one twin type to be treated per grain. Separate more phenomenological schemes are then introduced for representing the effects of these twin domains on the three mechanisms (i), (ii), and (iii) listed above.

Despite these advances, achieving a better understanding the nature of twinning in HCP requires more refined twinning models in combination with physically based models for dislocation slip. Unlike FCC or BCC metals, multiple twins are frequently observed in HCP metals, which depend on the distribution of crystallographic orientations and loading conditions. Specifically, more than one twin mode can form in a grain or one twin type can form within another twin type (secondary or internal twining). A good example of an HCP metal that exhibits 
multiple twinning modes as well as slip modes is $\mathrm{Zr}$. Four deformation twinning modes have been found in $\alpha-\mathrm{Zr}$ grains including the c-axis extension twins $\{10 \overline{1} 2\}\langle 10 \overline{1} \overline{1}\rangle$ and $\{11 \overline{2} 1\}\langle 10 \overline{2} \overline{6}\rangle$ and the c-axis contraction twins $\{11 \overline{2} 2\}\langle 11 \overline{2} \overline{3}\rangle$ and $\{10 \overline{1} 1\}\langle 10 \overline{1} \overline{2}\rangle$. In many instances, the former two tensile twins can exist in the same grain [31]. These twins re-reorient the Zr crystal lattice by $85.22^{\circ}$ about the $\langle 11 \overline{2} 0\rangle$ direction, $34.84^{\circ}$ about the $\langle 10 \overline{1} 0\rangle$ direction, $64.22^{\circ}$ about the $\langle 10 \overline{1} 0\rangle$ direction, and $57.05^{\circ}$ about the $\langle 11 \overline{2} 0\rangle$ direction, respectively [32, 33]. Because of these large reorientations, which twin modes are selected and the twin volume fractions have a profound influence on texture evolution and macroscopic strain hardening. In addition, primary twins can favorably reorient a crystal for secondary twinning. Secondary twinning can significantly alter the deformation and failure response of $\mathrm{Zr}$ [31].

In addition to twinning, $\alpha-\mathrm{Zr}$ is also known to generally deform by more than one slip mode. Through polycrystal and single crystal testing, prismatic $\{\overline{1} 100\}\langle\overline{1} \overline{1} 20\rangle$ slip has been established as the easiest glide mode in $\operatorname{Zr}[34,35]$. This has been attributed to its c/a ratio of 1.594, which is lower than the ideal c/a ratio of 1.633 . Pyramidal $\{10 \overline{1} 1\}\langle\overline{1} \overline{1} 23\rangle$ slip systems are harder than prismatic slip systems, but offer the additional degrees of freedom necessary to accommodate arbitrary plastic strains [36]. Basal $\{0001\}\langle\overline{1} \overline{1} 20\rangle$ slip also operates in Zr but requires higher activation stresses than the prismatic systems [37]. Moreover, these slip modes are highly rate sensitive and each has a different dependency on temperature and strain rate. For instance, pyramidal slip is much more rate sensitive than prismatic slip and basal slip is the most rate sensitive [35-38].

Like slip, temperature and strain rate are also known to affect deformation twinning in HCP polycrystals. However, the reasons underlying this apparent rate sensitivity of twinning are not as well understood as for slip. First of all, twinning often acts in combination with slip, and slip alone, as mentioned, is highly rate sensitive. Second, most of the foregoing studies on $\mathrm{Zr}$ (and other HCP metals) have analyzed slip and twinning within narrow windows of temperature or strain rates. Last, interpretation of relative contributions of various deformation modes is usually done based on the models that do not simultaneously consider the pronounced changes in texture and stress-strain evolution, and the underlying effects of strain rate and temperature on activation and propagation of individual deformation mechanisms.

In this work, we aim to understand the influence of strain rate and temperature on the activation of primary and secondary twinning modes in $\alpha$-Zr. In particular, we seek to uncover 
the role that dislocation slip and its rate sensitivity play on the types of primary and secondary twins activated. We use a comprehensive set of experimental data collected in the temperature range of $76-673 \mathrm{~K}$ and strain rate range of $0.001-4500 \mathrm{~s}^{-1}$ using standard and Split-Hopkinson Pressure Bar mechanical testing, electron backscattered diffraction (EBSD) and X-ray diffraction. The variety of testing conditions as well as the large strain deformation levels achieved ( $>30 \%$ strain) allowed us to examine rate dependencies of three slip modes: prismatic $<\mathrm{a}>\operatorname{slip}$ (easiest), pyramidal <c+a > slip (harder), and basal <a> slip (hardest) and three twinning modes. For inferring the underlying deformation mechanisms, we employ the visco-plastic selfconsistent (VPSC) model with a strain rate and temperature hardening law based on the explicit evolution of dislocation density to the material under investigation and lattice reorientation by primary and secondary twinning. The VPSC model is shown capable of reproducing both the stress-strain response and microstructure evolution for all test conditions using a single set of material parameters. The model predictions are used to determine the underlying deformation mechanisms and their effect on mechanical response and texture evolution in $\alpha$-Zr. We find that over the wide temperature and strain rate range tested the preferred tension twin is $\{10 \overline{1} 2\}\langle 10 \overline{1} \overline{1}\rangle$ twinning whether it is a primary or secondary twin. Also, as reported in prior single crystal experiments, we observe that the preferred compression twin changes from $\{11 \overline{2} 2\}\langle 11 \overline{2} \overline{3}\rangle$ to $\{10 \overline{1} 1\}\langle 10 \overline{1} \overline{2}\rangle$ as temperature increases. We additionally show that this transition is not sensitive to strain rate and whether the contraction twin is a primary or secondary twin. Based on the dominant mechanisms revealed in modeling and texture evolution, we rationalize the temperature-induced transition in contraction twinning is due to the increased activity of basal <a> slip at high temperatures (> 673K).

\subsection{Material and experimental methods}

\subsection{Material}

The material studied here is a clock-rolled and fully recrystallized plate of $\mathrm{Zr}$ with the chemical composition expressed in weight parts per million (Wt. Ppm) of: $\mathrm{O}<50, \mathrm{C}<22, \mathrm{~N}<$ 20, $\mathrm{Fe}<50, \mathrm{Al}<20, \mathrm{~V}<50, \mathrm{Hf} 35, \mathrm{Ti}<25$ and $\mathrm{Zr}$ balance. The texture and microstructure of the initial material were examined as part of an earlier study [39]. Electron backscattered diffraction (EBSD) and optical micrographs revealed a nearly equi-axed and twin-free grain structure with a mean grain size of $25 \mu \mathrm{m}$ (Fig. 1a). 
The initial crystallographic texture was measured by both X-ray diffraction (XRD) [39, 40] and EBSD over a large area and both measurements show that most of the basal poles are oriented within $20^{\circ}$ from the normal direction of the rolled sheet (the so-called strong basal texture). Since EBSD measures micro-texture and XRD measures macro-texture, we calculated the orientation distribution function (ODF) from the XRD data (using popLA software) and used it to generate the discrete texture file needed for the input initial texture for the crystal plasticity simulations reported here. The pole figures of the measured and converted textures are compared in Fig. 1b. Plotting was done in the MTEX software package [41]. As shown, they are very similar except the maximum intensity of the measured one is slightly higher. Evidently, the conversion from the measured texture into the polycrystal data file caused the loss of intensity from $\sim 5.2$ to 4.8 .

\subsection{Mechanical testing}

The mechanical response of the $\mathrm{Zr}$ plate was measured in compression and tension under quasi-static conditions and in compression under high-strain rate conditions. Compression samples were machined from the plate as cylinders $5 \mathrm{~mm}$ in diameter by $5 \mathrm{~mm}$ in length. The axis of the specimens was either aligned with the in-plane (IP samples) or through-thickness (TT samples) direction of the rolled $\mathrm{Zr}$ sheet. Tensile samples were machined as dogbone specimens with a $3.2 \mathrm{~mm}$ square cross-section. In what follows, these samples will be labeled as IPC (inplane compression), IPT (in-plane-tension), and TTC (through-thickness compression). Table 1 lists the testing conditions for each test. Constant velocity mechanical tests were performed using a screw-driven Instron. To reduce frictional effects during loading of the compression samples, we used polished tungsten carbide plates lubricated with molybdenum disulfide grease. The raw load-displacement data were collected and corrected for machine compliance before computing the true stress-true strain curves. Dynamic compression tests were performed using a SplitHopkinson Pressure Bar [42, 43]. The inherent oscillations in the dynamic stress-strain curves make the determination of the yield stress very approximate at low strains.

\subsection{EBSD characterization}

EBSD maps were measured in an FEI XL30 SEM equipped with TSL/EDAX data acquisition software. Following deformation, the samples were cut vertically in half with the section plane containing the loading direction. The specimens were prepared by grinding to an 800 grit $\mathrm{SiC}$ finish followed by chemically polishing with a solution of the ratio 
45H2O:45HNO3:10HF. Scans were taken at $20 \mathrm{kV}$ with a $0.2 \mu \mathrm{m}$ step size in a hexagonal grid. Twin statistics were analyzed using the OIM ${ }^{\mathrm{TM}}$ Analysis software and a twin program written at Los Alamos National Laboratory [31, 44].

\subsection{Multi-scale modeling approach}

In this section, we review the multi-scale modeling approach we employ to distill the individual dependencies of dislocation evolution, twin selection, and twin growth on strain rate and temperature. At the largest scale, we model the constitutive response of the polycrsytal and its relationship to the deformation of the individual constituent crystals via the viscoplastic selfconsistent (VPSC) scheme. At the grain scale, we use crystal plasticity for slip and a composite grain model for the lattice reorientation induced by the primary twins. At the subgrain scale, we introduce a thermally activated dislocation density law for the evolution of slip resistance for the individual slip systems as a function of temperature and strain rate. The barriers for twin activation and propagation within each crystal follow a different model than slip and this twinning model will be reviewed last. In several prior studies, this same basic modeling framework has been successfully applied to a variety of metals of different crystal structures: $\mathrm{Zr}$ [45, 46], Be [47, 48], and $\mathrm{Mg}$ [49, 50], BCC Ta [51-53], an FCC cobalt alloy [54], and orthorhombic uranium $[55,56]$. However, to meet the objectives of this work, we found it necessary to extend the framework to treat multiple modes of twins per crystal, interactions between slip modes, and lattice reorientation by secondary (internal) twinning. These additions will be highlighted in what follows.

\subsection{Visco-plastic self-consistent (VPSC) model}

In VPSC, a polycrystal is represented by a set of crystals or model grains (each having an orientation, shape and a volume fraction). Each grain is treated as a visco-plastic inclusion embedded in a homogeneous effective medium. The elementary inclusion problem is solved using a Green's function approach [23]. Enforcing the macro-homogeneity condition of selfconsistency between the macroscopic stress and grain average stresses allows for calculation of the viscoplastic properties of the effective medium. The effective medium response corresponds to that of the polycrystal and is to be compared with that of the tested sample.

Plasticity in each grain is carried via the activation of slip and twinning. The corresponding slip or twin shearing rate, $\dot{\gamma}^{s}$, on a given system, $s$, is modeled using the power-law relation: 


$$
\dot{\gamma}^{s}=\dot{\gamma}_{0}\left|\frac{\tau^{s}}{\tau_{c}^{s}}\right|^{\frac{1}{m}} \operatorname{sign}\left(\tau^{s}\right) .
$$

Here, $\dot{\gamma_{0}}$ is the reference slip rate set to be the macroscopic strain rate, $m$ is the rate sensitivity parameter set to be $0.05, \tau^{S}$ is the resolved shear stress, and $\tau_{c}^{s}$ is the threshold stress on the slip system. The twin propagation is treated as a pseudo-slip mechanism, as originally proposed in [11].

According to Eq. (1), the crystal plasticity constitutive law requires a threshold resistance to activate each slip mode and each twinning mode. In this modeling framework, these activation stresses can evolve with strain separately for each system within each grain in the polycrystal. Twin activation is modeled separately from slip and the expansion of twin domains may be indirectly affected by the stored dislocation density. The models related to slip are described next and those related to twinning as well as to the interactions between twins and slip dislocations are described in the next section.

\subsection{Dislocation density hardening law (DD model)}

For dislocation slip, resistances change as dislocations are stored in the crystal. The dislocation density for each slip system $s$ in each slip mode $\alpha$ evolves following its individual law and possesses its own dependencies on strain rate and temperature. We adopt a thermally

activated dislocation density based hardening law (DD model) that was developed previously $[50,57]$ and therefore, is only briefly reviewed below since the physically based parameters that the law uses (athermal stress, activation barriers, drag stress, etc.) are important for interpreting our experimental results. In the description that follows, the superscripts $s$ and $s$ span the slip systems and the Greek superscripts $\alpha, \beta$ represent modes to which $s$ and $s$ ' belong.

The resistance to slip $\tau_{c}^{s}$ includes a friction stress $\tau_{o}^{\alpha}$ (dependent on the Peierls stress and the initial content of dislocations), a barrier term $\tau_{o, H P}^{\alpha}$ (dependent on initial grain size) and two terms that evolve with strain, i.e., the forest and debris interaction stresses $\tau_{f o r}^{s}$ and $\tau_{d e b}^{\alpha}$ (dependent on a spatially random and ordered distribution of stored dislocations, respectively) $[45,58]$ :

$$
\tau_{c}^{s}=\tau_{o}^{\alpha}+\tau_{o, H P}^{\alpha}+\tau_{f o r}^{s}+\tau_{d e b}^{\alpha} .
$$


In this work, we are concerned with strain rate and temperature sensitive deformation. The initial slip resistances $\tau_{o}^{\alpha}(\dot{\varepsilon}, T)$ per slip mode are found to decay exponentially with temperature and increase logarithmically with strain rate as:

$$
\tau_{o}^{\alpha}(\dot{\varepsilon}, \mathrm{T})=A^{\alpha}\left(1+B^{\alpha} \log (\dot{\varepsilon})\right) \exp \left(-\frac{\mathrm{T}}{C^{\alpha}}\right)\left(1+D^{\alpha} \operatorname{lognormal}\left(\sigma^{\alpha}, v^{\alpha}\right)\right),
$$

where $A^{\alpha}, B^{\alpha}$, and $C^{\alpha}$ are constants and $\mathrm{T}$ and $\dot{\varepsilon}$ are the current temperature and strain rate, respectively. Such expressions were used for several other metals in earlier studies [5, 45, 54, 56, 59]. The constant $D^{\alpha}$ and lognormal terms, $\sigma^{\alpha}$ and $v^{\alpha}$, are used to capture the saturation of the resistances at high temperatures seen experimentally. The grain size barrier term, $\tau_{o, H P}^{\alpha}$, is formulated through a Hall-Petch like equation:

$$
\tau_{o, H P}^{\alpha}=\mu^{\alpha}(\mathrm{T}) H P^{\alpha} \sqrt{\frac{b^{\alpha}}{d_{g}}}
$$

where $b^{\alpha}$ is the magnitude of the Burgers vector, $d_{g}$ is the initial grain size, $\mu^{\alpha}(T)=40.06-0.022 \mathrm{~T}$ is the effective shear modulus taken to be the same for all shear directions [60], and $H P^{\alpha}$ is the Hall-Petch parameter. When twins are present in a grain, the $\tau_{o, H P}^{\alpha}$ term is defined per slip system, $s$, as $\tau_{H P}^{S}=\frac{f^{p t s}-f^{p t s, 0}}{f^{p t s, m a x}-f^{p t s, 0}} \mu^{\alpha}(\mathrm{T}) H P^{\alpha} \sqrt{\frac{b^{\alpha}}{d_{m f p}^{s}}}$ and $d_{g}$ is replaced by the mean-free-path distance calculated using $d_{m f p}^{s}=\frac{\left(1-f^{p t s}\right) d_{c}}{\sin (\alpha)}[7,45]$. The angle $\alpha$ is the angle between slip plane and the predominant twin system (PTS) plane and $d_{c}=\frac{d_{g}}{n^{\text {lamellas }}}$ is the separation between twin lamellae calculated as the ratio between the grain size, $d_{g}$, and a specified number of lamellae, $n^{\text {lamellas }}$, which is assigned a nominal value of 5 . The term, $\frac{f^{p t s}-f^{p t s, 0}}{f^{p t s, m a x}-f^{p t s, 0}}$, represents the barrier factor and is based on the $f^{p t s, 0}$ and $f^{p t s, m a x}$ constants, which represent the minimum volume fraction identifying the PTS and the maximum volume fraction that a twin can occupy within a given grain. The former $f^{p t s, 0}$ is taken to be 0.02 and the latter $f^{\text {pts,max }}$ is taken to be 0.9 . 
The evolution of $\tau_{\text {for }}^{s}(\dot{\varepsilon}, \mathrm{T})$ and $\tau_{d e b}^{\alpha}(\dot{\varepsilon}, \mathrm{T})$ are governed by the evolution of the forest $\rho_{\text {for }}^{s}(\dot{\varepsilon}, \mathrm{T})$ and debris $\rho_{\text {deb }}(\dot{\varepsilon}, \mathrm{T})$ dislocation densities, as described below. The effects are given by Taylor-type laws [61, 62]:

$$
\begin{aligned}
& \tau_{f o r}^{s}(\dot{\varepsilon}, \mathrm{T})=b^{\alpha} \mu^{\alpha}(\mathrm{T}) \sqrt{\chi^{s s^{\prime}} \rho_{f o r}^{s^{\prime}}(\dot{\varepsilon}, \mathrm{T})} \\
& \tau_{d e b}^{\alpha}(\dot{\varepsilon}, \mathrm{T})=k_{d e b} \mu^{\alpha}(\mathrm{T}) b^{\alpha} \sqrt{\rho_{d e b}(\dot{\varepsilon}, \mathrm{T})} \log \left(\frac{1}{b^{\alpha} \sqrt{\rho_{d e b}(\dot{\varepsilon}, \mathrm{T})}}\right)
\end{aligned}
$$

where $\chi^{s s^{\prime}}$ is a dislocation interaction matrix. Accurate characterization of this parameter would require specialized experiments and dislocation dynamics simulations, e.g., [63]. To eliminate any uncertainty that might be introduced by this parameter and to keep the number of fitting parameters minimal, we utilize only the diagonal terms $\left(s=s^{\prime}\right)$ and set them all to a typically used value of 0.81 [61]. However, in principle, it is important that the model is capable of accounting for dislocation-dislocation interactions via the off-diagonal components of the matrix.

The evolution of the stored forest density, $\rho_{f o r}^{s}(\dot{\varepsilon}, \mathrm{T})$, is governed by competition between the rate of storage and the rate of dynamic recovery [61, 64]:

$$
\frac{\partial \rho_{f o r}^{s}}{\partial \gamma^{s^{\prime}}}=\frac{\partial \rho_{\text {gen,for }}^{s}}{\partial \gamma^{s^{\prime}}}-\frac{\partial \rho_{r e m, f o r}^{s}}{\partial \gamma^{s^{\prime}}}=k_{1}^{s} \sqrt{\rho_{f o r}^{s}}-k_{2}^{s}(\dot{\varepsilon}, \mathrm{T}) \rho_{f o r}^{s} .
$$

Here, $k_{1}^{s}$ is a rate-insensitive coefficient for dislocation storage by statistical trapping of mobile dislocations and $k_{2}^{s}$ is a rate-sensitive coefficient that accounts for dynamic recovery by thermally activated mechanisms. Following the derivation given in [45], we define the coefficient, $k_{2}^{s}$, to include not only an averaged interaction coefficient $\chi$ but also the anisotropic interactions through $\chi^{s s^{\prime}}$ :

$$
\frac{k_{1}^{s}}{k_{2}^{s}}=\frac{1}{\sqrt{\left(\chi^{-1}\right)^{s^{\prime} s}\left(\frac{\tau_{\text {sat }}^{s}}{b^{\alpha} \mu^{\alpha}}\right)^{2}}}, \tau_{s a t}^{s}=\frac{D^{\alpha}\left(b^{\alpha}\right)^{3} g^{\alpha} \mu^{\alpha}}{D^{\alpha}\left(b^{\alpha}\right)^{3}-k \mathrm{~T} \ln \left(\frac{\dot{\varepsilon}}{\dot{\varepsilon}_{0}}\right)} .
$$

where $k, \dot{\varepsilon}_{o}, g^{\alpha}$, and $D^{\alpha}$ are the Boltzmann constant, a reference strain rate taken to be $10^{7} \mathrm{~s}^{-1}$, an effective activation enthalpy, and a drag stress, respectively. Dynamic recovery is often associated with thermal activation of dislocation cross-slip and climb, and the formation of 
dislocation debris is concomitant with recovery processes. The rate of debris development is coupled to the rate of recovery of all active dislocations through:

$$
d \rho_{d e b}=\sum_{s} q^{\alpha} b^{\alpha} \frac{\partial \rho_{r e m, f o r}^{s}}{\partial \gamma^{s^{\prime}}}\left|d \gamma^{s^{\prime}}\right|
$$

where $q^{\alpha}$ as a rate coefficient defining a fraction of dislocations that leads to debris formation and the remaining fraction that annihilates.

\subsection{Twin activation}

Twin nucleation occurs at the atomic length scale where a unique strength for forming a twin nucleus can be potentially calculated [65]. At a larger scale, such as the one we treat here, it is difficult to experimentally characterize a unique critical resolved shear stress for twinning [57]. The local stresses and defect structures from which twins arise are statistically varying [66]. Therefore, although we provide model parameters associated with twin formation, we cannot state that these are singular values tied directly to twin nucleation at the atomic scale. Despite these limitations, however, these model parameters provide insight into the relative propensity of twinning among the three twin types and three slip modes considered for $\mathrm{Zr}$ and the individual sensitivities of twinning to strain rate and temperature.

In the description that follows, we use $t$ to designate the twin variant and $\beta$ the twin mode to which $t$ belongs. The twin activation stress accounts for a temperature-independent friction term $\tau_{0}^{\beta}$, a Hall-Petch-like term $\tau_{o, H P}^{t}$, and a latent hardening term that couples slip and twin systems:

$$
\tau_{c}^{t}=\tau_{o}^{\beta}+\tau_{o, H P}^{t}+\tau_{s l i p}^{t} .
$$

The terms are defined as follows:

$$
\begin{aligned}
& \tau_{o}^{\beta}=\tau_{\text {prop }}^{\beta}+\left(\tau_{\text {crit }}^{\beta}-\tau_{\text {prop }}^{\beta}\right) \exp \left(-\frac{\sum \rho_{\text {for }}^{s}}{n^{\alpha} \rho_{\text {sat }}^{s}}\right), \\
& \tau_{o, H P}^{t}=\frac{H P^{\beta}}{\sqrt{d_{g}}},
\end{aligned}
$$

and

$$
\tau_{s l i p}^{t}=\mu^{\beta}(\mathrm{T}) \sum_{j} C^{\beta \alpha}(\dot{\varepsilon}) b^{\beta} b^{\alpha} \rho_{\text {for }}^{s}
$$


where $\tau_{\text {crit }}^{\beta}$ and $\tau_{\text {prop }}^{\beta}$ are the nucleation and propagation stresses, whose contribution to twin activation is leveraged by the probability term $\frac{\sum \rho_{\text {for }}^{s}}{n^{\alpha} \rho_{\text {sat }}^{s}}[45]$. The probability term is defined by an average value of dislocation density on slip systems, which is reflected in the division by $n^{\alpha}$, which is the number of slip systems in a given mode $\alpha$. For $\{10 \overline{1} 2\}\langle 10 \overline{1} \overline{1}\rangle$ and $\{11 \overline{2} 2\}\langle 11 \overline{2} \overline{3}\rangle$ twins, the dislocation density of prismatic slip systems enters the probability equation, whereas for $\{10 \overline{1} 1\}\langle 10 \overline{1} \overline{2}\rangle$ twins, the dislocation density of basal slip systems enters into the probability equation. Constants in the interaction term are the elastic shear modulus on the system, $\mu^{\beta}$, the Burgers vector of a given twin system, $b^{\beta}$, and the latent hardening matrix in function of strain rate, $C^{\beta \alpha}$. The six twin variants, $t$, belonging to the same twin mode, $\beta$, have their own activation stress and relationship to the dislocations stored within the crystal.

\subsection{Primary twinning}

Lattice re-orientation by twinning is modeled via the composite grain (CG) approach [7]. In this approach a twin system with the highest shear-rate among all active twin systems, i.e., the predominant twin system (PTS), is identified in a given grain. The selection criterion for the twin variant is not entirely deterministic. Specifically we use the following expression: $0.2 f^{t}+0.8 \cdot r \cdot f^{p t s, 0} \geq f^{p t s, 0}$, where $r$ is sampled from uniform distribution and ranges between 0 and $1, f^{t}$ is the twin volume fraction of the $t^{\text {th }}$ twin system and $f^{p t s, 0}$ is the threshold defined earlier. The first twin variant that satisfies this condition is selected and is the termed the "predominant twin system. The grain is then partitioned into stacks of flat ellipsoids having the crystallographic orientation of the predominant twin and the matrix grain. The short axis of the parent and twin ellipsoids is perpendicular to the twin plane. Twin growth is modeled by volume fraction transfer from the parent grain to the twin domain. As the ellipsoids representing the twins thicken, the ones representing the parent shrink.

We note that the PTS criterion is a simplifying assumption because it neglects many of the factors that are believed to contribute to twin variant selection. Several experimental studies have shown that a non-negligible fraction of the twins do not correspond to the variant with the highest Schmid factor. Several reasons have been proposed, such as those related to grain-grain interactions, local grain boundary defects, parent grain properties, neighboring grain properties, slip-twin and twin-twin transmission across grain boundaries, and stress fluctuations at grain 
boundaries [67-71]. Experiments and numerical simulations in [67, 69] have studied the role that strain accommodation at grain boundaries play. Other studies have examined the relation between twin variant selection and the backstresses [70] or the work of accommodation due to reaction of the twinning shear induced in a neighboring grain [71]. Generally, accounting for such effects in twin formation within constitutive modeling require supplemental or integrated calculations of the local strains and stresses at and in the vicinity of the grain boundaries and the response of the neighboring grains. Such calculations are possible with full-field models based on finite elements $[17,72]$ or Green's functions [73] that consider explicitly the grain structure of the metal [74] but not the mean-field formulation adopted here. Nevertheless, to first order, prior work using mean-field models have demonstrated that assuming that the twins associated with the highest Schmid factor variant can lead to reasonable predictions for twin volume fraction and texture for many low-symmetry metals including $\mathrm{Zr}[7,23,45,46]$, Be [47, 48], Mg [49, 50, 75], Ti [6] and $\mathrm{U}[55,56]$. This success likely results because the thicker twins usually correspond to twins with the higher Schmid factor $[68,76]$.

\subsection{Secondary twinning}

As a necessary new feature, we introduce twin reorientation by secondary twinning. In the model we allow secondary twins to nucleate when a primary twin fraction in a given grain reaches a fraction of 0.5 . Nucleation and propagation of secondary twins is modeled in a similar manner as that of the primary twins, with the difference being that the role of the parent grain is replaced by a primary twin.

In the next section we calibrate and critically validate the model using a comprehensive set of rate and temperature sensitive experimental data for $\mathrm{Zr}$.

\subsection{Material parameter characterization}

To have a model capable of accurately describing the mechanical behavior and microstructure evolution over a broad range of temperatures, strain rates, and loading conditions requires making available the following deformation mechanisms: prismatic <a> slip, first-order pyramidal <c+a $>$ slip, basal <a> slip, $\{10 \overline{1} 2\}\langle 10 \overline{1} \overline{1}\rangle$ twinning, $\{11 \overline{2} 2\}\langle 11 \overline{2} \overline{3}\rangle$ twinning, and $\{10 \overline{1} 1\}\langle 10 \overline{1} \overline{2}\rangle$ twinning. The slip and twin activation models we employ introduce a number of physically based material parameters. Although we can build on parameters characterized in prior work, it is necessary to extend the characterization effort to ensure that the parameters apply at much higher temperatures and strain rates. Our approach is to obtain a single set of 
material parameters by only fitting the calculated stress-strain curves with the measured ones for all mechanical tests in this study. Other data reported here (texture, twin statistics, variant selection) are used for validation.

We should mention briefly, that for high-purity $\mathrm{Zr}$, some of these parameters have been characterized previously. They have been obtained for prismatic slip and pyramidal slip at low strain rates for low to moderate strain levels $(<30 \%)$, where basal slip does not contribute significantly [57] and additionally for basal slip for large strains (> 30\%), where the basal-slip contribution becomes non-negligible [46]. The parameters for $\{10 \overline{1} 2\}\langle 10 \overline{1} \overline{1}\rangle$ and $\{11 \overline{2} 2\}\langle 11 \overline{2} \overline{3}\rangle$ twinning have also been characterized previously for $\mathrm{Zr}$ at relatively low temperatures (76 $\mathrm{K}$ to $400 \mathrm{~K})$ at quasi-static strain rates $[45,66]$. However those for $\{10 \overline{1} 1\}\langle 10 \overline{1} \overline{2}\rangle$ twinning are not available and hence are newly determined here. In the modeling, we also permit de-twinning to occur [48, 77], but under the monotonic loading conditions studied here, we find that not surprisingly it was not activated.

The starting microstructure of the model polycrystal was constructed using the measured initial texture (Fig. 1b), represented by 2944 distinct orientations (grains) each with a corresponding volume fraction. Consistent with the initial grain structure seen in Fig. 1, we assigned the initial grain shape to be equi-axed and its diameter $d_{\mathrm{g}}$ to be $25 \mu \mathrm{m}$. In the simulations, we impose homogeneous boundary conditions corresponding to uniaxial compression or tension in the prescribed load direction (with respect to the initial texture) and traction-free surfaces in the other two orthogonal directions.

Figure 2 compares the measured and calculated stress-strain curves. We note that the calculations achieve agreement in both the flow stresses and hardening rates for the entire suite of tests. Table 2 lists the single set of material parameters corresponding to these calculations. In particular, Figure 3 compares the variation in the initial activation stresses, $\tau_{0}^{\alpha}$, with temperature for each slip mode with experimental data on single crystals found in the literature [35-37, 78]. Other relevant model outputs, such as slip and twin activity as a function of strain, strain rate, and temperature; texture evolution; and twin volume fraction, were not used for parameter characterization, and thus, are model predictions to be analyzed.

\subsection{Results}

\subsection{Influence of strain rate and temperature on tensile twin selection}


Of the seven observed twin types in HCP metals [1, 2, 79], three are tensile (or extension) twins in Zr. Tensile twins are thus named because they accommodate a tensile strain along the caxis. Accordingly tensile twins can form under tensile loading parallel to the c-axis. Compression perpendicular to the c-axis can also result in a positive resolved shear stress on many of the tensile twin variants, and hence tensile twinning is often observed for compressive loading perpendicular to the c-axis. In the present material, due to the strong initial texture (Fig. 1b), in-plane compression (IPC) is favorable for tensile twinning. Prior work carried out on $\mathrm{Zr}$ of similar composition and initial texture after IPC found that the preferred twin type is the $\{10 \overline{1} 2\}\langle 10 \overline{1} \overline{1}\rangle$ twin for a temperature range of $76 \mathrm{~K}$ to $298 \mathrm{~K}$ and quasi-static strain rates [31, 77]. For this material, Figure 4 shows the EBSD maps after compressive deformation at a) $76 \mathrm{~K}$ and b) $298 \mathrm{~K}$ at $0.001 \mathrm{~s}^{-1}$. In agreement, the $\{10 \overline{1} 2\}\langle 10 \overline{1} \overline{1}\rangle$ twin is the preferred tensile twin. A much smaller fraction of the $\{10 \overline{2} 1\}\langle 11 \overline{2} \overline{6}\rangle$ twins is also observed at $76 \mathrm{~K}$. The preferred tensile twin type at higher temperatures, however, is not known since, under commonly used quasistatic testing conditions, slip dominates over twinning as temperature increases. To explore the selection of tensile twin type in HCP $\mathrm{Zr}$ as a function of temperature, we simultaneously apply high strain rates to promote twinning at high temperatures and use the model to separate the effects of strain rate and temperature.

Figure $4 \mathrm{c}$ shows the microstructure after room temperature compression deformation at high rate $\left(3500 \mathrm{~s}^{-1}\right)$. Prior to deformation, the grains had orientations such that their $\left.<11-20\right\rangle$ and $<10$ 10> (green to blue), directions were nearly aligned with the compression direction. After deformation, the microstructure has been almost completely transformed to near [0001] orientations by $\{10 \overline{1} 2\}\langle 10 \overline{1} \overline{1}\rangle$ twins. Next, we varied the test temperature from $298 \mathrm{~K}$ to $673 \mathrm{~K}$ while keeping the strain rate high $\left(4500 \mathrm{~s}^{-1}\right)$. Figure $4 \mathrm{~d}$ shows the EBSD maps of the deformed sample at $673 \mathrm{~K}$. Again, $\{10 \overline{1} 2\}\langle 10 \overline{1} \overline{1}\rangle$ twins have nearly completely transformed the orientations of the grains. Considering all tests performed, the preferred tensile twin is the $\{10 \overline{1} 2\}\langle 10 \overline{1} \overline{1}\rangle$ twin for a broad range of temperature $(76 \mathrm{~K}$ to $673 \mathrm{~K})$ and rates $\left(10^{-3} \mathrm{~s}^{-1}\right.$ to $10^{3} \mathrm{~s}^{-}$ ${ }^{1}$ ). We do not see a temperature- or strain rate-induced transition in the type of tension twin that prevails. Shortly we will discuss tests to determine whether this preference persists when $\{10 \overline{1} 2\}\langle 10 \overline{1} \overline{1}\rangle$ twinning serves as a secondary twin.

Figure 5 and 6 compare measured and predicted texture evolution. It can be seen that texture evolution is predicted well. In particular, the texture reorientations in IPC after large strains and 
under high strain rates due to profuse twinning are also effectively captured (Fig. $5 \mathrm{c}$ and d). Under these conditions, the basal $\{0001\}$ poles have almost completely reoriented toward the compression direction.

\subsection{Deformation within $\{10 \overline{1} 2\}\langle 10 \overline{1} \overline{1}\rangle$ twins}

The presence of twins in the grain can cause a change in the deformation mechanisms. The twins introduce twin boundaries, which can serve as barriers to certain slip systems. The twins also form reoriented domains that can promote different slip or twin modes within the twin than were active in the original, untwinned grain (matrix) under the same imposed stress state. Particularly, within $\{10 \overline{1} 2\}\langle 10 \overline{1} \overline{1}\rangle$ twins, the c-axis reorients by $85.22^{\circ}$ and aligns close to the imposed compression axis (Fig. 5c and d). Consequently, to accommodate compression, the twin domains can deform by slip and/or contraction twinning. To examine the temperature- and strain-rate dependence of the deformation mechanisms within the $\{10 \overline{1} 2\}\langle 10 \overline{1} \overline{1}\rangle$ twins, we examined the large-strain behavior under two conditions where profuse primary $\{10 \overline{1} 2\}\langle 10 \overline{1} \overline{1}\rangle$ twinning occurs: a) in-plane compression at cold temperature $(76 \mathrm{~K})$ and quasi-static strain rates and b) in-plane compression at high temperature and high strain rates $\left(>10^{3} \mathrm{~s}^{-1}\right)$.

For the first case a), Fig. 4a shows the microstructure after large strain at $0.001 \mathrm{~s}^{-1}$ and $76 \mathrm{~K}$. By a strain of 0.08 , secondary $\{11 \overline{2} 2\}\langle 11 \overline{2} \overline{3}\rangle$ twins form within the primary twins. This result is similar to that reported previously [31]. With the present model, we predict that the $\{10 \overline{1} 2\}\langle 10 \overline{1} \overline{1}\rangle$ twin domains deform primarily by $\{11 \overline{2} 2\}\langle 11 \overline{2} \overline{3}\rangle$ twinning at $76 \mathrm{~K}$ (see Fig. 7b). Both microstructural characterization and the model find that the volume fraction of secondary $\{11 \overline{2} 2\}\langle 11 \overline{2} \overline{3}\rangle$ twinning reaches around $3 \%$ at a strain of 0.18 and increases with further straining (see Fig. 9).

For the second case b), Fig. 4d shows the microstructure after the same deformation mode, in plane compression (IPC), but at a higher rate and higher temperature $\left(673 \mathrm{~K}\right.$ and $\left.4500 \mathrm{~s}^{-1}\right)$. Here we find that the $\{10 \overline{1} 2\}\langle 10 \overline{1} \overline{1}\rangle$ twins deform by different mechanisms than at colder temperatures and slower rates. We observe that secondary twinning occurs via another HCP contraction twin, the $\{10 \overline{1} 1\}\langle 10 \overline{1} \overline{2}\rangle$ twin, and not by the $\{11 \overline{2} 2\}\langle 11 \overline{2} \overline{3}\rangle$ twin. EBSD analysis and the model find that the volume fraction of secondary $\{10 \overline{1} 1\}\langle 10 \overline{1} \overline{2}\rangle$ twinning reaches approximately $7 \%$ by a strain of 0.44 . Thus, it appears that the choice of compression twin type as a secondary twin within the primary $\{10 \overline{1} 2\}\langle 10 \overline{1} \overline{1}\rangle$ twins exhibits a rate sensitivity. 
To determine whether the twin-type selection was caused by the increase in strain rate or in temperature, we tested the material in IPC at room temperature and high rate $\left(3500 \mathrm{~s}^{-1}\right)$. Figure $4 \mathrm{c}$ shows the deformed microstructure from EBSD. As shown, the high rate was sufficient to activate profuse primary $\{10 \overline{1} 2\}\langle 10 \overline{1} \overline{1}\rangle$ twinning. More importantly, secondary contraction twinning occurred via $\{11 \overline{2} 2\}\langle 11 \overline{2} \overline{3}\rangle$ twinning. The volume fraction of secondary twinning was estimated to be $6 \%$ at a strain of 0.32 .

Therefore, we see an interesting dependence of contraction twin type on temperature, not seen in the selection of the tensile twin type. With the same loading state, similar high-rate conditions, and the same primary $\{10 \overline{1} 2\}\langle 10 \overline{1} \overline{1}\rangle$ twin type, it appears that the preferred contraction twin type changes from $\{11 \overline{2} 2\}\langle 11 \overline{2} \overline{3}\rangle$ twinning at low temperatures (room temperature and lower) to $\{10 \overline{1} 1\}\langle 10 \overline{1} \overline{2}\rangle$ twinning at high temperatures $(673 \mathrm{~K})$.

This phenomenon has been reported in earlier work on single crystals of $\mathrm{Ti}$ and $\mathrm{Zr}$, which were compressed along the c-axis at different temperatures and low strain rates $[36,80]$. It was seen in Ti that below $673 \mathrm{~K}$, primary $\{11 \overline{2} 2\}\langle 11 \overline{2} \overline{3}\rangle$ twinning was favored and above it, primary $\{10 \overline{1} 1\}\langle 10 \overline{1} \overline{2}\rangle$ twinning was favored [80]. In single crystal $\mathrm{Zr}$, the transition occurs at $800 \mathrm{~K}$ [36]. The present results on polycrystalline $\mathrm{Zr}$ show that a similar transition occurs within primary twin domains as well and around $673 \mathrm{~K}$ (and possibly lower).

\subsection{Temperature sensitive contraction twin selection}

An understanding of twin selection among the various HCP twin types has yet to be developed [2]. For instance, it is not known why $\{11 \overline{2} 2\}\langle 11 \overline{2} \overline{3}\rangle$ contraction twins are preferred at lower temperatures and $\{10 \overline{1} 1\}\langle 10 \overline{1} \overline{2}\rangle$ twins at higher temperatures in transition metals such as $\mathrm{Ti}$ and $\mathrm{Zr}$ and not in Mg. Many studies have speculated that the answers lie in how each twin nucleates. As mentioned earlier in the context of tensile twinning, some nucleation mechanisms proposed for deformation twinning involve special boundary defect structures or slip dislocationboundary interactions $[81,82]$. With this in mind, it could be surmised that the transition in contraction twin type reported here may be the result of interactions of impinging slip dislocations from either the parent matrix or twin domain with the primary $\{10 \overline{1} 2\}\langle 10 \overline{1} \overline{1}\rangle$ twin boundary. This possibility leads us to the question: Is the observed transition in contraction twin type from $\{11 \overline{2} 2\}\langle 11 \overline{2} \overline{3}\rangle$ twinning at low temperatures to $\{10 \overline{1} 1\}\langle 10 \overline{1} \overline{2}\rangle$ twinning at high temperatures limited to secondary twinning? To test whether contraction twinning exhibits the 
same temperature dependence as a primary twin, we carried out uniaxial compression tests through- thickness of the rolled sheet (TTC tests).

At a low temperature of $76 \mathrm{~K}$, Figure $2 \mathrm{~d}$ shows the stress-strain response for TTC at two rates, $0.001 \mathrm{~s}^{-1}$ and $2000 \mathrm{~s}^{-1}$. Both exhibit similar responses characteristic of deformation dominated by twinning in that the hardening rate is constant or increases with strain. As evidence, figure $4 \mathrm{~g}$ shows the EBSD micrograph at $76 \mathrm{~K}$ and low rate $\left(0.001 \mathrm{~s}^{-1}\right)$ after 0.08 strain, where the microstructure is populated by $\{11 \overline{2} 2\}\langle 11 \overline{2} \overline{3}\rangle$ twins.

To activate twinning at higher temperatures, high-rate TTC was needed. Figure $2 \mathrm{e}$ shows the stress-strain curves at $298 \mathrm{~K}$ and at different rates: $0.001 \mathrm{~s}^{-1}, 0.1 \mathrm{~s}^{-1}$, and $2700 \mathrm{~s}^{-1}$. Only at the highest rate does the material exhibit a deformation response characteristic of $\{11 \overline{2} 2\}\langle 11 \overline{2} \overline{3}\rangle$ twinning. Therefore, $\{11 \overline{2} 2\}\langle 11 \overline{2} \overline{3}\rangle$ twinning is preferred at low temperatures and for high rates at room temperature.

To explore even higher temperatures and high rates, Fig. $2 \mathrm{f}$ compares the TTC response at $473 \mathrm{~K}$ at $3000 \mathrm{~s}^{-1}$ and $673 \mathrm{~K}$ at $3500 \mathrm{~s}^{-1}$. The former curve does not suggest substantial amounts of twinning occurred, but the latter one does. We see in Figure 4h, which is the EBSD micrograph at $673 \mathrm{~K}$ at high rate $\left(3500 \mathrm{~s}^{-1}\right)$ after 0.34 strain, that the deformed structure is dense with $\{10 \overline{1} 1\}\langle 10 \overline{1} \overline{2}\rangle$ twins. Compared to the initial texture the deformation textures in Fig. $6 \mathrm{~b}$ show an increased intensity in the basal poles near the TT direction due to basal slip activity, as will be discussed later. It is sufficient to counteract the effect of compressive twinning, which tends to diminish the intensity of the basal poles in the TT direction. In this case, the basal poles of the twinned domains reorient by $\{10 \overline{1} 1\}\langle 10 \overline{1} \overline{2}\rangle$ twinning $\left(57.05^{\circ}\right.$ away from the compression axis). The reorientation does not produce increased intensity near the perimeter of the basal pole figure for several reasons. First, the volume fraction of primary twins is relatively low. Second the twin orientations are distributed for 360 degrees about the perimeter and thus do not produce a large increase in intensity at any specific spot around the perimeter. Finally, there is also some reorientation due to $\{10 \overline{1} 2\}\langle 10 \overline{1} \overline{1}\rangle$ secondary twinning $\left(85.22^{\circ}\right.$ back toward the compression axis).

These results indicate that contraction twinning in $\mathrm{Zr}$, as a primary twin, has the same temperature dependence as it does as a secondary twin: $\{11 \overline{2} 2\}\langle 11 \overline{2} \overline{3}\rangle$ contraction twinning is the preferred twin mode at room temperature (and high rate) and below and $\{10 \overline{1} 1\}\langle 10 \overline{1} \overline{2}\rangle$ contraction twinning is preferred at the higher temperature, $673 \mathrm{~K}$. Therefore, it cannot be 
concluded that the temperature-induced transition in contraction twin type is solely related to the primary $\{10 \overline{1} 2\}\langle 10 \overline{1} \overline{1}\rangle$ twin domain or requires interactions only with the $\{10 \overline{1} 2\}\langle 10 \overline{1} \overline{1}\rangle$ twin boundary.

The domains of contraction twins are reoriented well for strain accommodation by tensile twinning. Characterization of both the $\{11 \overline{2} 2\}\langle 11 \overline{2} \overline{3}\rangle$ and $\{10 \overline{1} 1\}\langle 10 \overline{1} \overline{2}\rangle$ twinned structures in Fig. $7 \mathrm{a}$ and $7 \mathrm{~b}$, respectively, reveal $\{10 \overline{1} 2\}\langle 10 \overline{1} \overline{1}\rangle$ secondary twins. Thus, like $\{10 \overline{1} 2\}\langle 10 \overline{1} \overline{1}\rangle$ primary twinning, $\{10 \overline{1} 2\}\langle 10 \overline{1} \overline{1}\rangle$ secondary twinning also persists over a wide range of temperatures.

\subsection{Discussion}

6.1 Model interpretations on the influence of strain rate and temperature on the formation and deformation of twins

In this work, the model was used to interpret the mechanisms underlying the evolution of stress and texture during straining.

In all cases of temperature and strain rate, the IPC stress-strain curves exhibit typical signatures of twinning, where the work hardening rate is constant or increases with strain. The details in the shape of the deformation response, however, depend on the relative amounts of twinning and slip. HCP deformation textures are highly dependent on the relative contributions of slip and twinning as well as the type of twin and twin variants selected. Accordingly, texture can serve as an excellent signature of the active deformation mechanisms as well as a stringent test of our multi-scale model predictions. Figure 5 compares the calculated texture with the measurement for the IPC tests in Fig. 2. We can see that good agreement has been achieved.

From the model, we can extract predictions of the contributions of slip and twinning to deformation. Figure 8 shows the evolution of the relative activities of slip and twinning in the grains and in the twin domains corresponding to the IPC stress-strain responses. As a reference point, we begin with the case of ambient conditions (low rate and room temperature), where there is little twinning (Fig. 8a). The corresponding measured and predicted textures at a strain of 0.065 are provided in Fig. 5b. Both textures appear similar to the starting texture (Fig. 1b) due to the little reorientation due to twinning and predominance of prismatic slip.

At a lower temperature, $76 \mathrm{~K}$, the model predicts an increasing evolution of twin volume fraction with IPC strain, which quickly becomes profuse (50\% volume fraction) by a strain of 0.2 (Fig. 8b). We validate this prediction in Fig. 10, which compares the calculated and measured 
strain evolution of the twin volume fraction. Part of the measured data points are taken from [31]. Likewise, at higher rates $\left(3500 \mathrm{~s}^{-1}\right)$, the model indicates that twinning increases with IPC strain and eventually becomes profuse reaching $50 \%$ volume fraction by a strain of 0.14 (Fig. $8 \mathrm{c}$ ). At room temperature and high strain rate $\left(3500 \mathrm{~s}^{-1}\right)$ twinning is also profuse (Fig. $\left.8 \mathrm{~d}\right)$. We compare the calculated and measured textures at 0.32 strain in Fig. 5c. As shown, the texture has completely transformed by twinning and the basal poles have reoriented close to the compression direction.

For high strain rates and higher temperatures (473 K and $673 \mathrm{~K}$ ), the model continues to predict increasing tensile twin fractions with strain (Fig. 8e and f). The textures corresponding to $673 \mathrm{~K}$ are shown in Fig. 5d and appear, to first order, similar to those in Fig. 5c; that is, a texture indicative of profuse $\{10 \overline{1} 2\}\langle 10 \overline{1} \overline{1}\rangle$ twinning.

In the TTC tests, twinning did not occur in every condition tested. Figure 6 compares the calculated and measured textures and Fig. 9 presents the predicted slip and twinning activities. As a reference, little to no twinning is observed at quasi-static rates and room temperature and caxis compression deformation is accommodated by pyramidal slip. The model generally finds and, most importantly, quantifies that the relative amount of twinning relative to slip increases with lower temperatures $(76 \mathrm{~K})$ and higher strain rates $\left(>3500 \mathrm{~s}^{-1}\right)$. We validate the $76 \mathrm{~K}$ prediction in Fig. 11, which compares the calculated and measured strain evolution of the twin volume fraction in the TT compression. Regardless of strain rate, for temperatures $473 \mathrm{~K}$ and below, the model predicts that $\{11 \overline{2} 2\}\langle 11 \overline{2} \overline{3}\rangle$ twinning is preferred over $\{10 \overline{1} 1\}\langle 10 \overline{1} \overline{2}\rangle$ twinning. It is not until $673 \mathrm{~K}$ that $\{10 \overline{1} 1\}\langle 10 \overline{1} \overline{2}\rangle$ twinning occurs.

\subsection{Is twinning rate sensitive?}

In testing $\mathrm{Zr}$ over a wide range of loading conditions, we observe that strain rate and temperature strongly influence the amount and type of twin mode selected. However, these results do not necessarily imply that contraction and extension twinning possess individual dependencies on strain rate and temperature. As we have shown, the material stress-strain responses and associated texture evolution arise either from slip or from a combination of slip and twinning. Slip alone, both the amount and type of slip mode activated, is known to be highly sensitive to strain rate and temperature $[32,45,48,79]$. Thus, it is possible that the observed rate sensitivity in the macroscopic responses is a consequence of the rate sensitivities of the slip modes activated. 
With the model, we can de-convolute the macroscopic response into the individual rate sensitivities of slip and twinning. We can readily achieve this by examining the material parameters characterized by the model (Table 3). As shown in Table 3, the characteristic activation stresses for all three twinning modes used in the model are rate independent. Thus, the strain rate and temperature dependencies of stress-strain, twin volume fraction, texture evolution, and even the transition in contraction twin type captured by the model can be explained entirely by the strain rate and temperature dependence of slip.

According to the model, the easiest to form (lowest $\tau_{c r i t}^{\beta}$ ) and most mobile (lowest $\tau_{\text {prop }}^{\beta}$ ) is the $\{10 \overline{1} 2\}\langle 10 \overline{1} \overline{1}\rangle$ twin. This result is in complete agreement with expectation. Its low activation barrier can be attributed to the fact that this twin has the smallest Burgers vector and twin shear $S$. The topologies and kinetics of these twins have been studied using atomistic simulations [81, 83, 84]. With molecular dynamics (MD), it has been predicted that the $\{10 \overline{1} 2\}\langle 10 \overline{1} \overline{1}\rangle$ twin is more glissile than the other two twins $\{10 \overline{1} 1\}\langle 10 \overline{1} \overline{2}\rangle$ and $\{11 \overline{2} 2\}\langle 11 \overline{2} \overline{3}\rangle$ $[81,85]$.

The model finds that the $\{10 \overline{1} 1\}\langle 10 \overline{1} \overline{2}\rangle$ twin is the hardest to activate and the $\{11 \overline{2} 2\}\langle 11 \overline{2} \overline{3}\rangle$ twin is the hardest to propagate (highest $\tau_{\text {prop }}^{\beta}$ ). This trend can be linked to basic topological properties of the respective twinning dislocations for the twin types. The former has the highest twin shear and the twinning dislocation of the latter has the largest number of layers, with three layers as opposed to two $[85,86]$.

Establishing the rate sensitivity of twinning is directly relevant to understanding the effect of temperature on contraction twinning. Both earlier work on single crystals [36] and the present work on polycrystals find that contraction twinning in $\mathrm{Zr}$ exhibits a temperature-induced transition from $\{11 \overline{2} 2\}\langle 11 \overline{2} \overline{3}\rangle$ twinning at lower temperatures and $\{10 \overline{1} 1\}\langle 10 \overline{1} \overline{2}\rangle$ twinning at higher temperatures. To date, the reason for the transition has yet to be clarified. It was first proposed that twinning is rate sensitive, which would imply that the "CRSS" for $\{11 \overline{2} 2\}\langle 11 \overline{2} \overline{3}\rangle$ twinning increased with temperature and $\{10 \overline{1} 1\}\langle 10 \overline{1} \overline{2}\rangle$ twinning decreased with temperature [80], but this explanation assumes the ideal case in which slip dislocations were not active or contributing to deformation. Later, using a homogeneous twin nucleation model, it was argued that $\{11 \overline{2} 2\}\langle 11 \overline{2} \overline{3}\rangle$ twinning is preferred over $\{10 \overline{1} 1\}\langle 10 \overline{1} \overline{2}\rangle$ twinning when the twin-boundary energy for $\{10 \overline{1} 1\}\langle 10 \overline{1} \overline{2}\rangle$ twins is within $10 \%$ of or greater than that for $\{11 \overline{2} 2\}\langle 11 \overline{2} \overline{3}\rangle$ twins 
and that transition arises because their relative boundary energies change with temperature [87]. However, both MD and first principles calculations find that the $\{10 \overline{1} 1\}\langle 10 \overline{1} \overline{2}\rangle$ twin boundary energy is substantially lower than the $\{11 \overline{2} 2\}\langle 11 \overline{2} \overline{3}\rangle$ twin boundary energy [88-91], with 74.3 $\mathrm{mJ} / \mathrm{m}^{2}$ compared to $123.8 \mathrm{~mJ} / \mathrm{m}^{2}$. Likewise, differences in the mobility of $\{10 \overline{1} 1\}\langle 10 \overline{1} \overline{2}\rangle$ and $\{11 \overline{2} 2\}\langle 11 \overline{2} \overline{3}\rangle$ twinning dislocations cannot provide an explanation [90]. Atomic-scale simulations show that the $\{10 \overline{1} 1\}\langle 10 \overline{1} \overline{2}\rangle$ twin is the more mobile twin [81]. Last, as we have just discussed, the present model calculations indicate that twinning is rate insensitive and the rateinsensitive activation parameters found for these three twin types are consistent with atomistic calculations.

Based on the above discussion, we hypothesize that the transition in contraction twinning with temperature is a consequence of slip. Slip assists in the formation of twins $[1,2,67,82,92-$ 94] and thus, when slip is altered by temperature, twinning would then be altered by temperature. To this end, we discuss next the rate sensitivity of slip suggested by the model before returning to the issue of the temperature-induced transition on contraction twinning.

\subsection{Special twin and slip combinations}

An interesting finding made by the model is the differences in the choice of slip mode activated between the high- and low-temperature conditions. Unlike twinning, results on slip are not apparent from EBSD microstructure characterization or readily obvious from texture analysis and thus the model plays an important role in uncovering the effects of rate on the relative contributions of the different slip systems.

In IPC, regardless of rate, the model indicates that tensile twinning is accompanied by prismatic slip at room and lower temperatures, and by a mix of prismatic and basal slip at higher temperatures (Fig. 8). Likewise, the twin domains deform predominantly by pyramidal slip and $\{11 \overline{2} 2\}\langle 11 \overline{2} \overline{3}\rangle$ (secondary) twinning at lower temperatures (including room temperature) and basal slip and $\{10 \overline{1} 1\}\langle 10 \overline{1} \overline{2}\rangle$ (secondary) twinning at higher temperatures. The former finding has been reported previously for polycrystalline $\mathrm{Zr}$ [31], but not the latter.

In TTC, a similar coupling of slip and twin systems is also seen. Figures 9 show the relative slip activities calculated by the model for the selected TTC tests. As temperature increases from $76 \mathrm{~K}$ to room temperature, the deformation mechanisms change from prismatic <a> slip and $\{11 \overline{2} 2\}\langle 11 \overline{2} \overline{3}\rangle$ twinning to prismatic $<\mathrm{a}>$ and pyramidal $<\mathrm{c}+\mathrm{a}>\operatorname{slip}$ (Fig. 8a). As temperature increases further to $473 \mathrm{~K}$, prismatic < a $>$ and pyramidal <c+a> slip still prevail, but basal <a> 
slip begins to contribute (Fig. 9e). At $673 \mathrm{~K}$, basal <a> slip, some pyramidal <c+a> slip and $\{10 \overline{1} 1\}\langle 10 \overline{1} \overline{2}\rangle$ twinning accommodate the deformation (Fig. 9f). Again, we see that at high temperatures, basal <a> slip and $\{10 \overline{1} 1\}\langle 10 \overline{1} \overline{2}\rangle$ twinning occur concomitantly.

The paired twin-slip mechanisms at higher temperatures (basal slip and $\{10 \overline{1} 1\}\langle 10 \overline{1} \overline{2}\rangle$ twinning) predicted by the model are supported by the good agreement achieved in the flow stress-strain curve for different test conditions (Fig. 2). Reinforcement for this prediction can also be found in subtle but important features of texture development. Consider the texture in Fig. 5d, measured at $673 \mathrm{~K}$ after high rate deformation to a strain of 0.44 . With modeling we can correlate features of this texture with the occurrence of basal slip within both the matrix and twin domains of the twinned grains. Figure 12 shows textures resulting from simulations a) with basal slip suppressed, b) with basal slip only within the matrix, and c) basal slip only within the $\{10 \overline{1} 1\}\langle 10 \overline{1} \overline{2}\rangle$ twin domains. By comparing a) and b), we see that allowing for basal slip to occur in the matrix stabilizes those twin domains that have reoriented the c-axis by $\sim 64^{\circ}$ from the TT compression direction, again consistent with the measurement. Comparing a) and c), we find that permitting basal slip to occur within the twinned domains reorients some crystals towards the IP1 axis as shown experimentally. Clearly allowing for basal slip to activate in both the matrix and twin as in the calculation of Fig. $5 \mathrm{~d}$ best explains the texture development seen experimentally. It is another piece of evidence to support that basal slip and $\{10 \overline{1} 1\}\langle 10 \overline{1} \overline{2}\rangle$ twinning are both associated with high temperatures, but not lower ones.

\subsection{Basal slip at high temperatures}

In this work, we report evidence of uncommonly large activity of basal slip in high rate, high temperature conditions. Analysis of flow stresses (Fig. 2) and details of texture development (Fig. 5d) together strongly indicate that basal slip prevails in both the grains and twin domains. Prior joint experimental and modeling studies have shown that basal slip in $\mathrm{Zr}$ is the hardest of the three common slip modes in HCP metals (prismatic, pyramidal, and basal) to activate. In low strain, ambient conditions, basal slip is not activated in single crystals [37], and typically occurs only at localized regions of the microstructure, such as near cracks [32, 37], near twins and kink bands [95], and free surfaces [37, 96-98]. Anomalously high contributions of basal slip in $\mathrm{Zr}$ have been reported in ambient conditions in large strain and severe plastic deformation, e.g., > 1.0 [46, 99], which are much higher than the strain levels achieved here. Recent ab initio calculations reveal that basal slip in $\mathrm{Zr}$ involves the thermally activated motion of a prismatic 
stacking fault normal to the prismatic plane [100], implying that high temperatures would promote basal slip. Consistent with these prior works, the present model finds that the activation stresses for basal slip below $673 \mathrm{~K}$ are significantly higher than those for prismatic and pyramidal slip (see Table 2).

The reliability of our finding on the prevalence of basal slip at high temperatures depends on the model's ability to capture the temperature dependence of the other slip modes. Thus, we seek to validate our activation stresses used by the model with available experimental data. Most of the data use single crystals and test for the rate and temperature dependence of prismatic <a> slip, which the easiest and most prevalent slip systems in $\mathrm{Zr}$ [35-37, 78]. Figure 3 compares the model values for the initial activation stresses for prismatic slip with the CRSS values reported in these works. Both the measured CRSS and the model $\tau_{0}^{\alpha}$ are intended to be a characteristic threshold stress that is separate from the effect of grain boundaries and dislocation-dislocation interactions. As shown, the measured CRSS for prismatic <a> slip drastically decreases as temperature increases and notably, the model $\tau_{0}^{\alpha}$ varies with temperature in a similar way.

Measurements are not available for the full range of temperatures tested here for pyramidal $<\mathrm{c}+\mathrm{a}>$ slip and basal <a> slip in $\mathrm{Zr}$, and therefore, we include experimental data outside the range in $\mathrm{Zr}$ and within the range from other HCP metals. For pyramidal $<\mathrm{c}+\mathrm{a}>\mathrm{slip}$, a strong temperature-dependent behavior in the CRSS has been reported for single crystals of Co and Be [101], which respectively have c/a ratios higher and lower than $\mathrm{Zr}$. To compare with the model pyramidal $<\mathrm{c}+\mathrm{a}>\operatorname{slip} \tau_{0}^{\alpha}$ for $\mathrm{Zr}$, we considered the Co measurements and reduced all the values consistently by 2.5 times. As Fig. $3 \mathrm{~b}$ shows, the temperature dependence is captured well by the model values for the pyramidal $<\mathrm{c}+\mathrm{a}>\operatorname{slip} \tau_{0}^{\alpha}$. (Co exhibits an anomalous increase in CRSS at high temperature from $\sim 400 \mathrm{~K}$ to $600 \mathrm{~K}$ that we do not forecast for $\mathrm{Zr}$.) For basal <a> slip, the model comparison is limited since basal slip activity was not detected in single crystals of $\mathrm{Zr}$ until above $800 \mathrm{~K}$ [37]. As seen in Fig. 3c, the model predicts a reduction in basal slip $\tau_{0}^{\alpha}$ to values in agreement with the measurement as temperature increases. The favorable comparison made implies prevalence of basal slip in the high temperature-high rate observations can be explained by a significant reduction in the CRSS at high temperatures.

6.5 The role of slip in the $\{11 \overline{2} 2\}\langle 11 \overline{2} \overline{3}\rangle-\{10 \overline{1} 1\}\langle 10 \overline{1} \overline{2}\rangle$ twin transition 
To date, the mechanisms underlying twin nucleation remain elusive. While it is possible to observe the formation of a twin in-situ, it is not possible to see the atomic scale details of the nucleation mechanism $[102,103]$. Throughout the years dislocation theory and atomic-scale simulations have been used and many of the more recent efforts are confirming some of the earlier notions that twinning results from slip dislocations [81, 82, 92]. Mendelson proposed that twinning dislocations could be produced from non-planar dissociations of slip dislocations [86, 93]. Based on detailed TEM analyses, Vaidya and Mahajan proposed that dislocations within slip bands can create embryonic $\{10 \overline{2} 1\}\langle 10 \overline{2} \overline{6}\rangle$ twins [94]. Yoo proposed several slip-twin boundary interactions that would support twin thickening [82]. In studying the events leading up to twin nucleation for different HCP twin types, MD simulations have supported many of these ideas [2, $65,81,104-106]$. On this basis, the origin of the $\{11 \overline{2} 2\}\langle 11 \overline{2} \overline{3}\rangle-\{10 \overline{1} 1\}\langle 10 \overline{1} \overline{2}\rangle$ twin temperature-induced transition could be a direct consequence of the temperature dependence of slip. In all tests of loading direction and strain rate, a predominance of prismatic <a> slip occurs at low temperatures (below $673 \mathrm{~K}$ ) when $\{11 \overline{2} 2\}\langle 11 \overline{2} \overline{3}\rangle$ the twin prevails and basal <a> slip at higher temperatures when the $\{10 \overline{1} 1\}\langle 10 \overline{1} \overline{2}\rangle$ twin is preferred.

A possible link between prismatic slip and the $\{11 \overline{2} 2\}\langle 11 \overline{2} \overline{3}\rangle$ twin and basal slip and the $\{10 \overline{1} 1\}\langle 10 \overline{1} \overline{2}\rangle$ twin has been made in a prior modeling study, which investigated the mechanism of twin nucleation via non-planar dissociations of <a> slip dislocations from either the basal or prismatic planes onto seven possible twinning planes [67]. Using continuum dislocation theory, a simple analytical model was developed that considered the energy and stability of the extended core of the twinning dislocation after the dissociation to determine whether slip-assisted twin nucleation was feasible. It was found that a suitable size dislocation pile-up was necessary but not sufficient to form a stable twin fault by a non-planar dissociation of an <a> slip dislocation. The calculations revealed that elastic dislocation interactions were a key factor for twinning. Specifically when the interaction force between the slip and twinning dislocations is repulsive, a pile-up, or any suitable defect arrangement, can potentially lead to a twin formation, whereas with no such repulsive force, no value of twin fault energy or pile up size can produce a twin. For $\mathrm{Zr}$, the calculations indicated that pile-ups of prismatic $<\mathrm{a}>$ dislocations can trigger a nonplanar dissociation reaction of the lead prismatic dislocation that produces twinning dislocations for $\{11 \overline{2} 2\}\langle 11 \overline{2} \overline{3}\rangle$ twins but not for $\{10 \overline{1} 1\}\langle 10 \overline{1} \overline{2}\rangle$ twins. However pile-ups of basal <a> dislocations can result in the non-planar dissociation of the lead basal dislocation that produces 
$\{10 \overline{1} 1\}\langle 10 \overline{1} \overline{2}\rangle$ twinning dislocations. On this basis, it appears the substantially enhanced basal slip activity at high temperatures may have promoted the formation of $\{10 \overline{1} 1\}\langle 10 \overline{1} \overline{2}\rangle$ contraction twins over $\{11 \overline{2} 2\}\langle 11 \overline{2} \overline{3}\rangle$ twins. On this basis, one would expect that $\{10 \overline{1} 1\}\langle 10 \overline{1} \overline{2}\rangle$ compression twinning would be profuse in materials where basal slip is predominant such as in Mg alloys. However $\{10 \overline{1} 1\}\langle 10 \overline{1} \overline{2}\rangle$ twins in Mg alloys tend to be very fine since they readily form internal $\{10 \overline{1} 2\}\langle 10 \overline{1} \overline{1}\rangle$ twins (a double twin sequence) $[3,104,107]$. Consequently they appear in low volume fractions, and are hard to detect in texture measurements.

We note that although providing a plausible reason, this idea only considers twin formation via a non-planar dissociation mechanism. Other dissociation types as well as other slip-assisted twin nucleation mechanisms may operate as well and these may have different dependencies on temperature. For instance, it is possible that dissociations from $\langle\mathrm{c}\rangle$ or $\langle\mathrm{c}+\mathrm{a}\rangle$ dislocations can produce twinning dislocations $[86,93,104]$.

\subsection{Conclusions}

In this work, we use a combination of experiment and multi-scale modeling to study the influence of strain rate and temperature on the activation of primary and secondary twinning modes in HCP $\alpha-Z r$. Numerous mechanical tests and subsequent characterization are carried out at different loading directions, strain rates, and temperatures. The model predictions achieve good agreement with measurements of twin-type selection and twin volume fraction with a single material parameter set. The model shows that the relative contributions of prismatic $<a>$, pyramidal $\langle\mathrm{c}+\mathrm{a}\rangle$, and basal $<\mathrm{a}\rangle$ slip and $\{10 \overline{1} 2\}\langle 10 \overline{1} \overline{1}\rangle,\{11 \overline{2} 2\}\langle 11 \overline{2} \overline{3}\rangle$, and $\{10 \overline{1} 1\}\langle 10 \overline{1} \overline{2}\rangle$ twinning to strain accommodation depend strongly on strain rate and temperature. These results can notably be explained by rate-independent twin activation and rate-dependent slip. We find that the $\{10 \overline{1} 2\}\langle 10 \overline{1} \overline{1}\rangle$ twin is favored over a wide range of temperatures ( $76 \mathrm{~K}$ to $673 \mathrm{~K}$ ). In contrast, we show that the preferred contraction twin depends on temperature with $\{11 \overline{2} 2\}\langle 11 \overline{2} \overline{3}\rangle$ twinning at lower temperatures and $\{10 \overline{1} 1\}\langle 10 \overline{1} \overline{2}\rangle$ twinning at higher temperatures. Moreover, these preferences are found to be insensitive to whether the twin is a primary twin or secondary internal twin and thus are not a special consequence of interactions with a pre-existing twin boundary. We rationalize that the preferences are likely a result of the slip systems activated. Based on dislocation theory, it was found previously that dissociations of the lead dislocation in a basal dislocation pile-up, but not a prismatic dislocation pile up in $\mathrm{Zr}$ can result in twinning dislocations for $\{10 \overline{1} 1\}\langle 10 \overline{1} \overline{2}\rangle$ twins. This concept suggests that the 
favorability of $\{10 \overline{1} 1\}\langle 10 \overline{1} \overline{2}\rangle$ twinning at high temperatures is likely linked to the emergence of prevalent basal slip at high temperature.

\section{Acknowledgements}

This work was performed under the subcontract No. 277871 granted by Los Alamos National Laboratory and the CMMI grant No. 1301081 from the National Science Foundation. IJB and RJM were supported by the Los Alamos National Laboratory Directed Research and Development (LDRD) project 20140348ER. JFB acknowledges the Joint DoD/DOE Munitions Technology Program for support.

\section{References}

[1] J.W. Christian, S. Mahajan. Deformation twinning, Progress in Materials Science 39 (1995) 1-157.

[2] I.J. Beyerlein, X. Zhang, A. Misra. Growth Twins and Deformation Twins in Metals, Annual Review of Materials Research 44 (2014) 329-363.

[3] M. Knezevic, A. Levinson, R. Harris, R.K. Mishra, R.D. Doherty, S.R. Kalidindi. Deformation twinning in AZ31: Influence on strain hardening and texture evolution, Acta Materialia 58 (2010) 6230-6242.

[4] S. Asgari, E. El-Danaf, S.R. Kalidindi, R.D. Doherty. Strain hardening regimes and microstructural evolution during large strain compression of low stacking fault energy fcc alloys that form deformation twins, Metallurgical and Materials Transactions A: Physical Metallurgy and Materials Science 28A (1997) 1781-1795.

[5] M. Knezevic, L. Capolungo, C.N. Tomé, R.A. Lebensohn, D.J. Alexander, B. Mihaila, R.J. McCabe. Anisotropic stress-strain response and microstructure evolution of textured $\alpha$ uranium, Acta Materialia 60 (2012) 702-715.

[6] M. Knezevic, R.A. Lebensohn, O. Cazacu, B. Revil-Baudard, G. Proust, S.C. Vogel, M.E. Nixon. Modeling bending of $\alpha$-titanium with embedded polycrystal plasticity in implicit finite elements, Materials Science and Engineering: A 564 (2013) 116-126.

[7] G. Proust, C.N. Tomé, G.C. Kaschner. Modeling texture, twinning and hardening evolution during deformation of hexagonal materials, Acta Materialia 55 (2007) 2137-2148.

[8] S. Bouvier, N. Benmhenni, W. Tirry, F. Gregory, M.E. Nixon, O. Cazacu, L. Rabet. Hardening in relation with microstructure evolution of high purity $\alpha$-titanium deformed under monotonic and cyclic simple shear loadings at room temperature, Materials Science and Engineering: A 535 (2012) 12-21.

[9] G.C. Kaschner, C.N. Tomé, R.J. McCabe, A. Misra, S.C. Vogel, D.W. Brown. Exploring the dislocation/twin interactions in zirconium, Materials Science and Engineering: A 463 (2007) 122-127.

[10] Z.S. Basinski, M.S. Szczerba, M. Niewczas, J.D. Embury, S.J. Basinski. Transformation of slip dislocations during twinning of copper-aluminum alloy crystals, Revue de Metallurgie. Cahiers D'Informations Techniques 94 (1997) 1037-1044.

[11] P. Van Houtte. Simulation of the rolling and shear texture of brass by the Taylor theory adapted for mechanical twinning, Acta Metallurgica et Materialia 26 (1978) 591-604. 
[12] C.N. Tomé, R.A. Lebensohn, U.F. Kocks. A model for texture development dominated by deformation twinning: Application to zirconium alloys, Acta Metallurgica et Materialia 39 (1991) 2667-2680.

[13] X. Wu, S.R. Kalidindi, C. Necker, A.A. Salem. Prediction of crystallographic texture evolution and anisotropic stress-strain curves during large plastic strains in high purity $\alpha$ titanium using a Taylor-type crystal plasticity model, Acta Materialia 55 (2007) 423-432.

[14] T. Fast, M. Knezevic, S.R. Kalidindi. Application of microstructure sensitive design to structural components produced from hexagonal polycrystalline metals, Computational Materials Science 43 (2008) 374-383.

[15] G.I. Taylor. Plastic strain in metals, Journal of the Institute of Metals 62 (1938) 307-324.

[16] M. Knezevic, S.R. Kalidindi, D. Fullwood. Computationally efficient database and spectral interpolation for fully plastic Taylor-type crystal plasticity calculations of face-centered cubic polycrystals, International Journal of Plasticity 24 (2008) 1264-1276.

[17] S.R. Kalidindi, H.K. Duvvuru, M. Knezevic. Spectral calibration of crystal plasticity models, Acta Materialia 54 (2006) 1795-1804.

[18] J.B. Shaffer, M. Knezevic, S.R. Kalidindi. Building texture evolution networks for deformation processing of polycrystalline fcc metals using spectral approaches: Applications to process design for targeted performance, International Journal of Plasticity 26 (2010) 1183-1194.

[19] M. Knezevic, H.F. Al-Harbi, S.R. Kalidindi. Crystal plasticity simulations using discrete Fourier transforms, Acta Materialia 57 (2009) 1777-1784.

[20] M. Knezevic, D.J. Savage. A high-performance computational framework for fast crystal plasticity simulations, Computational Materials Science 83 (2014) 101-106.

[21] B. Mihaila, M. Knezevic, A. Cardenas. Three orders of magnitude improved efficiency with high - performance spectral crystal plasticity on GPU platforms, International Journal for Numerical Methods in Engineering 97 (2014) 785-798.

[22] H.F. Al-Harbi, M. Knezevic, S.R. Kalidindi. Spectral Approaches for the Fast Computation of Yield Surfaces and First-Order Plastic Property Closures for Polycrystalline Materials with Cubic-Triclinic Textures, CMC: Computers, Materials, \& Continua 15 (2010) 153-172.

[23] R.A. Lebensohn, C.N. Tomé. A self-consistent anisotropic approach for the simulation of plastic deformation and texture development of polycrystals: Application to zirconium alloys, Acta Metallurgica et Materialia 41 (1993) 2611-2624.

[24] R.A. Lebensohn, C.N. Tomé, P.P. Castaneda. Self-consistent modelling of the mechanical behaviour of viscoplastic polycrystals incorporating intragranular field fluctuations, Philosophical Magazine 87 (2007) 4287-4322.

[25] M. Knezevic, M. Jahedi, Y.P. Korkolis, I.J. Beyerlein. Material-based design of the extrusion of bimetallic tubes, Computational Materials Science 95 (2014) 63-73.

[26] S.R. Kalidindi, C.A. Bronkhorst, L. Anand. Crystallographic Texture Evolution in Bulk Deformation Processing of Fcc Metals, Journal of the Mechanics and Physics of Solids 40 (1992) 537-569.

[27] R.J. Asaro, A. Needleman. Texture development and strain hardening in rate dependent polycrystals, Acta Metallurgica et Materialia 33 (1985) 923-953.

[28] X. Wu, G. Proust, M. Knezevic, S.R. Kalidindi. Elastic-plastic property closures for hexagonal close-packed polycrystalline metals using first-order bounding theories, Acta Materialia 55 (2007) 2729-2737. 
[29] M. Knezevic, S.R. Kalidindi. Fast computation of first-order elastic-plastic closures for polycrystalline cubic-orthorhombic microstructures, Computational Materials Science 39 (2007) 643-648.

[30] M. Knezevic, S.R. Kalidindi, R.K. Mishra. Delineation of first-order closures for plastic properties requiring explicit consideration of strain hardening and crystallographic texture evolution, International Journal of Plasticity 24 (2008) 327-342.

[31] R.J. McCabe, G. Proust, E.K. Cerreta, A. Misra. Quantitative analysis of deformation twinning in zirconium, International Journal of Plasticity 25 (2009) 454-472.

[32] M.H. Yoo. Slip, twinning, and fracture in hexagonal close-packed metals, Metall. Mater. Trans. A 12 (1981) 409-418.

[33] E. Tenckhoff. Deformation mechanisms, texture, and anisotropy in zirconium and zircaloy, ASTM International, 1988.

[34] A. Akhtar. Schmid's law and prismatic slip of zirconium, Scripta Metallurgica 9 (1975) 859-861.

[35] A. Akhtar. Prismatic slip in zirconium single crystals at elevated temperatures, Metallurgical Transactions A 6 (1975) 1217-1222.

[36] A. Akhtar. Compression of zirconium single crystals parallel to the c-axis, Journal of Nuclear Materials 47 (1973) 79-86.

[37] A. Akhtar. Basal slip in zirconium, Acta Metallurgica 21 (1973) 1-11.

[38] D. Mills, G. Craig. The plastic deformation of zirconium-oxygen alloy single crystals in the range 77 to $950 \mathrm{~K}$, TRANS MET SOC AIME 242 (1968).

[39] J. Bingert, T. Mason, G. Kaschner, G. Gray III, P. Maudlin. Deformation twinning in polycrystalline Zr: Insights from electron backscattered diffraction characterization, Metallurgical and Materials Transactions A 33 (2002) 955-963.

[40] M. Jahedi, M.H. Paydar, S. Zheng, I.J. Beyerlein, M. Knezevic. Texture evolution and enhanced grain refinement under high-pressure-double-torsion, Materials Science and Engineering: A 611 (2014) 29-36.

[41] F. Bachmann, R. Hielscher, H. Schaeben. Texture analysis with MTEX-free and open source software toolbox, Solid State Phenomena 160 (2010) 63-68.

[42] P.S. Follansbee. High Strain Rate Compression Testing - The Hopkinson Bar. 9th edn. Vol. 8, Am. Soc. Metals, Metals Park, Ohio, 1985. p.198-203.

[43] S.R. Chen, U.F. Kocks. High-Temperature Plasticity in Copper Polycrystals, High Temperature Constitutive Modeling - Theory and Application. A.D. Freed and K.P. Walker, eds., Atlanta, GA, The American Society of Mechanical Engineers, 1991. p.1-12.

[44] P. Marshall, G. Proust, J. Rogers, R. McCabe. Automatic twin statistics from electron backscattered diffraction data, Journal of microscopy 238 (2010) 218-229.

[45] I.J. Beyerlein, C.N. Tomé. A dislocation-based constitutive law for pure Zr including temperature effects, International Journal of Plasticity 24 (2008) 867-895.

[46] M. Knezevic, I.J. Beyerlein, T. Nizolek, N.A. Mara, T.M. Pollock. Anomalous Basal Slip Activity in Zirconium under High-strain Deformation, Materials Research Letters 1 (2013) 133140.

[47] D.W. Brown, I.J. Beyerlein, T.A. Sisneros, B. Clausen, C.N. Tomé. Role of twinning and slip during compressive deformation of beryllium as a function of strain rate, International Journal of Plasticity 29 (2012) 120-135. 
[48] M. Knezevic, I.J. Beyerlein, D.W. Brown, T.A. Sisneros, C.N. Tomé. A polycrystal plasticity model for predicting mechanical response and texture evolution during strain-path changes: Application to beryllium, International Journal of Plasticity 49 (2013) 185-198.

[49] A.L. Oppedal, H. El Kadiri, C.N. Tomé, G.C. Kaschner, S.C. Vogel, J.C. Baird, M.F. Horstemeyer. Effect of dislocation transmutation on modeling hardening mechanisms by twinning in magnesium, International Journal of Plasticity 30-31 (2012) 41-61.

[50] I.J. Beyerlein, R.J. McCabe, C.N. Tomé. Effect of microstructure on the nucleation of deformation twins in polycrystalline high-purity magnesium: A multi-scale modeling study, Journal of the Mechanics and Physics of Solids 59 (2011) 988-1003.

[51] M. Knezevic, T. Nizolek, M. Ardeljan, I.J. Beyerlein, N.A. Mara, T.M. Pollock. Texture evolution in two-phase $\mathrm{Zr} / \mathrm{Nb}$ lamellar composites during accumulative roll bonding, International Journal of Plasticity 57 (2014) 16-28.

[52] M. Ardeljan, I.J. Beyerlein, M. Knezevic. A dislocation density based crystal plasticity finite element model: Application to a two-phase polycrystalline HCP/BCC composites, Journal of the Mechanics and Physics of Solids 66 (2014) 16-31.

[53] M. Knezevic, I.J. Beyerlein, M.L. Lovato, C.N. Tomé, A.W. Richards, R.J. McCabe. A strain-rate and temperature dependent constitutive model for BCC metals incorporating nonSchmid effects: Application to tantalum-tungsten alloys, International Journal of Plasticity 62 (2014) 93-104.

[54] M. Knezevic, J.S. Carpenter, M.L. Lovato, R.J. McCabe. Deformation behavior of the cobalt-based superalloy Haynes 25: Experimental characterization and crystal plasticity modeling, Acta Materialia 63 (2014) 162-168.

[55] M. Knezevic, R.J. McCabe, R.A. Lebensohn, C.N. Tomé, C. Liu, M.L. Lovato, B. Mihaila. Integration of self-consistent polycrystal plasticity with dislocation density based hardening laws within an implicit finite element framework: Application to low-symmetry metals, Journal of the Mechanics and Physics of Solids 61 (2013) 2034-2046.

[56] M. Knezevic, R.J. McCabe, C.N. Tomé, R.A. Lebensohn, S.R. Chen, C.M. Cady, G.T. Gray Iii, B. Mihaila. Modeling mechanical response and texture evolution of $\alpha$-uranium as a function of strain rate and temperature using polycrystal plasticity, International Journal of Plasticity 43 (2013) 70-84.

[57] I.J. Beyerlein, R.J. McCabe, C.N. Tome. Stochastic processes of $\{1012\}$ deformation twinning in hexagonal close-packed polycrystalline zirconium and magnesium, International Journal for Multiscale Computational Engineering 9 (2011) 459-480.

[58] R. Madec, B. Devincre, L. Kubin, T. Hoc, D. Rodney. The role of collinear interaction in dislocation-induced hardening, Science 301 (2003) 1879-1882.

[59] P.W. Flynn, J. Motte, J.E. Dorn. On the Thermally Activated. Mechanism of Prismatic Slip in Magnesium Single Crystals, Trans Metall Soc AIME 221 (1961) 1148-1154.

[60] E. Fisher, C. Renken. Single-crystal elastic moduli and the hcp $\rightarrow$ bcc transformation in Ti, Zr, and Hf, Physical Review 135 (1964) A482.

[61] H. Mecking, U.F. Kocks. Kinetics of flow and strain-hardening., Acta Metallurgica et Materialia 29 (1981) 1865-1875.

[62] R. Madec, B. Devincre, L.P. Kubin. From Dislocation Junctions to Forest Hardening, Physical Review Letters 89 (2002) 255508.

[63] N. Bertin, C. Tomé, I. Beyerlein, M. Barnett, L. Capolungo. On the strength of dislocation interactions and their effect on latent hardening in pure Magnesium, International Journal of Plasticity 62 (2014) 72-92. 
[64] U. Essmann, H. Mughrabi. Annihilation of dislocations during tensile and cyclic deformation and limits of dislocation densities, Philosophical Magazine A 40 (1979) 731-756.

[65] J. Wang, S. Yadav, J. Hirth, C. Tomé, I. Beyerlein. Pure-shuffle nucleation of deformation twins in hexagonal-close-packed metals, Materials Research Letters 1 (2013) 126132.

[66] S.R. Niezgoda, A.K. Kanjarla, I.J. Beyerlein, C.N. Tomé. Stochastic modeling of twin nucleation in polycrystals: An application in hexagonal close-packed metals, International Journal of Plasticity 56 (2014) 119-138.

[67] L. Capolungo, I. Beyerlein. Nucleation and stability of twins in hcp metals, Physical Review B 78 (2008) 024117.

[68] I. Beyerlein, L. Capolungo, P. Marshall, R. McCabe, C. Tomé. Statistical analyses of deformation twinning in magnesium, Philosophical Magazine 90 (2010) 2161-2190.

[69] L. Capolungo, I.J. Beyerlein, G.C. Kaschner, C.N. Tomé. On the interaction between slip dislocations and twins in HCP Zr, Materials Science and Engineering: A 513-514 (2009) 42-51.

[70] B. Clausen, C.N. Tomé, D.W. Brown, S.R. Agnew. Reorientation and stress relaxation due to twinning: Modeling and experimental characterization for $\mathrm{Mg}$, Acta Materialia 56 (2008) 2456-2468.

[71] S. Mu, J.J. Jonas, G. Gottstein. Variant selection of primary, secondary and tertiary twins in a deformed Mg alloy, Acta Materialia 60 (2012) 2043-2053.

[72] F. Roters, P. Eisenlohr, L. Hantcherli, D.D. Tjahjanto, T.R. Bieler, D. Raabe. Overview of constitutive laws, kinematics, homogenization and multiscale methods in crystal plasticity finite-element modeling: Theory, experiments, applications, Acta Materialia 58 (2010) 11521211.

[73] R.A. Lebensohn, A.K. Kanjarla, P. Eisenlohr. An elasto-viscoplastic formulation based on fast Fourier transforms for the prediction of micromechanical fields in polycrystalline materials, International Journal of Plasticity 32-33 (2012) 59-69.

[74] M. Knezevic, B. Drach, M. Ardeljan, I.J. Beyerlein. Three dimensional predictions of grain scale plasticity and grain boundaries using crystal plasticity finite element models, Computer Methods in Applied Mechanics and Engineering 277 (2014) 239-259.

[75] G. Proust, C.N. Tomé, A. Jain, S.R. Agnew. Modeling the effect of twinning and detwinning during strain-path changes of magnesium alloy AZ31, International Journal of Plasticity 25 (2009) 861-880.

[76] L. Capolungo, P. Marshall, R. McCabe, I. Beyerlein, C. Tomé. Nucleation and growth of twins in Zr: a statistical study, Acta Materialia 57 (2009) 6047-6056.

[77] G. Proust, G.C. Kaschner, I.J. Beyerlein, B. Clausen, D.W. Brown, R.J. McCabe, C.N. Tomé. Detwinning of High-Purity Zirconium: In-Situ Neutron Diffraction Experiments, Experimental Mechanics 50 (2010) 125-133.

[78] A. Akhtar, A. Teghtsoonian. Plastic deformation of zirconium single crystals, Acta Metallurgica 19 (1971) 655-663.

[79] P.G. Partridge. The crystallography and deformation modes of hexagonal close-packed metals, Metallurgia Revised 12 (1967) 169.

[80] N.E. Paton, W.A. Backofen. Plastic deformation of titanium at elevated temperatures, Metallurgical Transactions 1 (1970) 2839-2847.

[81] A. Serra, R.C. Pond, D.J. Bacon. Computer simulation of the structure and mobility of twinning disclocations in H.C.P. Metals, Acta Metallurgica et Materialia 39 (1991) 1469-1480. 
[82] M.H. Yoo. Interaction of slip dislocations with twins in HCP metals, Trans. Met. Soc. AIME 245 (1969) 2051-2060.

[83] A. Serra, D. Bacon. Computer simulation of twin boundaries in the hcp metals, Philosophical Magazine A 54 (1986) 793-804.

[84] J. Wang, R.G. Hoagland, J.P. Hirth, L. Capolungo, I.J. Beyerleinb, C.N. Tome. Nucleation of a (1012) twin in hexagonal close-packed crystals, Scripta Materialia 61 (2009) 903-906.

[85] J. Wang, I.J. Beyerlein, J.P. Hirth, C.N. Tomé. Twinning dislocations on $\{1011\}$ and $\{1013\}$ planes in hexagonal close-packed crystals, Acta Materialia 59 (2011) 3990-4001.

[86] S. Mendelson. Dislocation dissociations in hcp metals, Journal of Applied Physics 41 (1970) 1893-1910.

[87] M. Yoo, J. Lee. Deformation twinning in hep metals and alloys, Philosophical Magazine A 63 (1991) 987-1000.

[88] J. Wang, I.J. Beyerlein. Atomic Structures of [0110] Symmetric Tilt Grain Boundaries in Hexagonal Close-Packed (hcp) Crystals, Metallurgical and Materials Transactions A 43 (2012) 3556-3569.

[89] J. Wang, I.J. Beyerlein. Atomic structures of symmetric tilt grain boundaries in hexagonal close packed (hcp) crystals, Modelling and Simulation in Materials Science and Engineering 20 (2012) 024002.

[90] J. Morris, Y. Ye, K. Ho, C. Chan, M. Yoo. A first-principles study of compression twins in hcp zirconium, Philosophical magazine letters 69 (1994) 189-195.

[91] R. Bauer, E.A. Jägle, W. Baumann, E.J. Mittemeijer. Kinetics of the allotropic hcp-fcc phase transformation in cobalt, Philosophical Magazine 91 (2011) 437-457.

[92] S. Mahajan. Critique of mechanisms of formation of deformation, annealing and growth twins: Face-centered cubic metals and alloys, Scripta Materialia 68 (2013) 95-99.

[93] S. Mendelson. Zonal dislocations and twin lamellae in h.c.p. metals, Materials Science and Engineering 4 (1969) 231-242.

[94] S. Vaidya, S. Mahajan. Accommodation and formation of $\{1121\}$ twins in Co single crystals, Acta Metallurgica 28 (1980) 1123-1131.

[95] J.L. Martin, R.E. Reed-Hill. A Study of Basal Slip Kink Bands in Polycrystalline Zirconium, Transactions of the Metallurgical Society of AIME 230 (1964) 780-785.

[96] J.I. Dickson, G.B. Craig. Room-temperature basal slip in zirconium, Journal of Nuclear Materials 40 (1971) 346-348.

[97] H. Francillette, B. Bacroix, M. Gaspérini, J.L. Béchade. Effect of initial textures on deformation mechanisms and texture evolutions of Zr $\alpha$ polycrystals deformed by channel-die compression tests, Materials Science and Engineering: A 234-236 (1997) 974-977.

[98] H. Francillette, B. Bacroix, M. Gaspérini, J.L. Béchade. Grain orientation effects in Zr702 $\alpha$ polycrystalline samples deformed in channel die compression at room temperature, Acta Materialia 46 (1998) 4131-4142.

[99] G.G. Yapici, C.N. Tomé, I.J. Beyerlein, I. Karaman, S.C. Vogel, C. Liu. Plastic flow anisotropy of pure zirconium after severe plastic deformation at room temperature, Acta Materialia 57 (2009) 4855-4865.

[100] N. Chaari, E. Clouet, D. Rodney. First-Principles Study of Secondary Slip in Zirconium, Physical review letters 112 (2014) 075504.

[101] A. Akhtar. Pyramidal slip in cobalt, Scripta Metallurgica 10 (1976) 365-366. 
[102] S.J. Zheng, I.J. Beyerlein, J. Wang, J.S. Carpenter, W.Z. Han, N.A. Mara. Deformation twinning mechanisms from bimetal interfaces as revealed by in situ straining in the TEM, Acta Materialia 60 (2012) 5858-5866.

[103] J. Ye, R.K. Mishra, A.K. Sachdev, A.M. Minor. In situ TEM compression testing of Mg and Mg-0.2 wt.\% Ce single crystals, Scripta Materialia 64 (2011) 292-295.

[104] I. Beyerlein, J. Wang, M. Barnett, C. Tomé. Double twinning mechanisms in magnesium alloys via dissociation of lattice dislocations, Proceedings of the Royal Society A: Mathematical, Physical and Engineering Science 468 (2012) 1496-1520.

[105] Y. Minonishi, S. Ishioka, M. Koiwa, S. Mobozumi. The structure of $\{1121\}$ twin boundaries in HCP crystals, physica status solidi (a) 71 (1982) 253-258.

[106] B. Xu, L. Capolungo, D. Rodney. On the importance of prismatic/basal interfaces in the growth of (1012) twins in hexagonal close packed crystals, Scripta Materialia 68 (2013) 901904.

[107] M.R. Barnett. Twinning and the ductility of magnesium alloys: Part II. "Contraction" twins, Materials Science and Engineering: A 464 (2007) 8-16. 


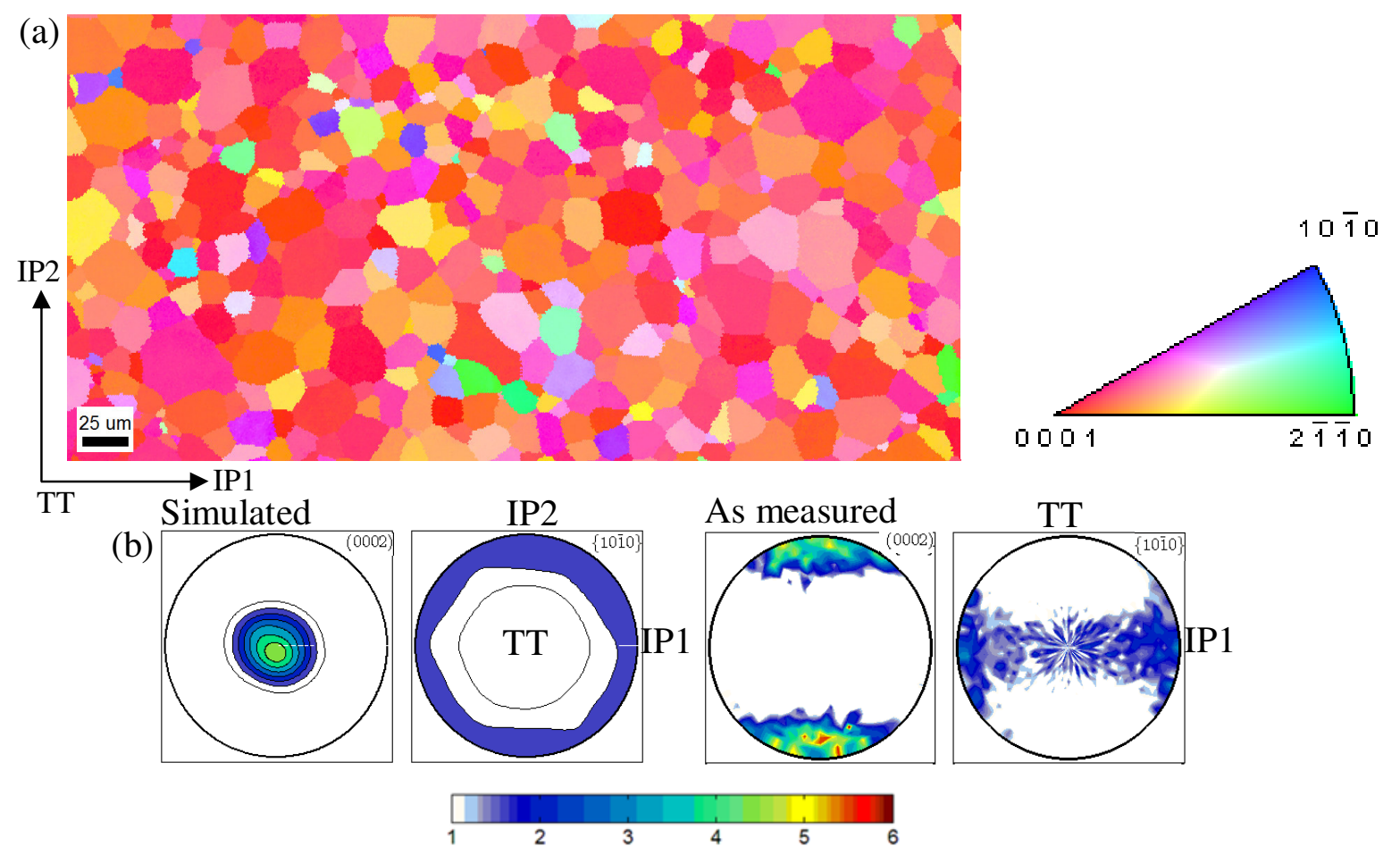

Figure 1 (a) An orientation map and (b) the pole figures of the initial microstructure and texture of the clock-rolled and recrystallized zirconium plate. The initial texture has been measured by $\mathrm{X}$-ray diffraction. 

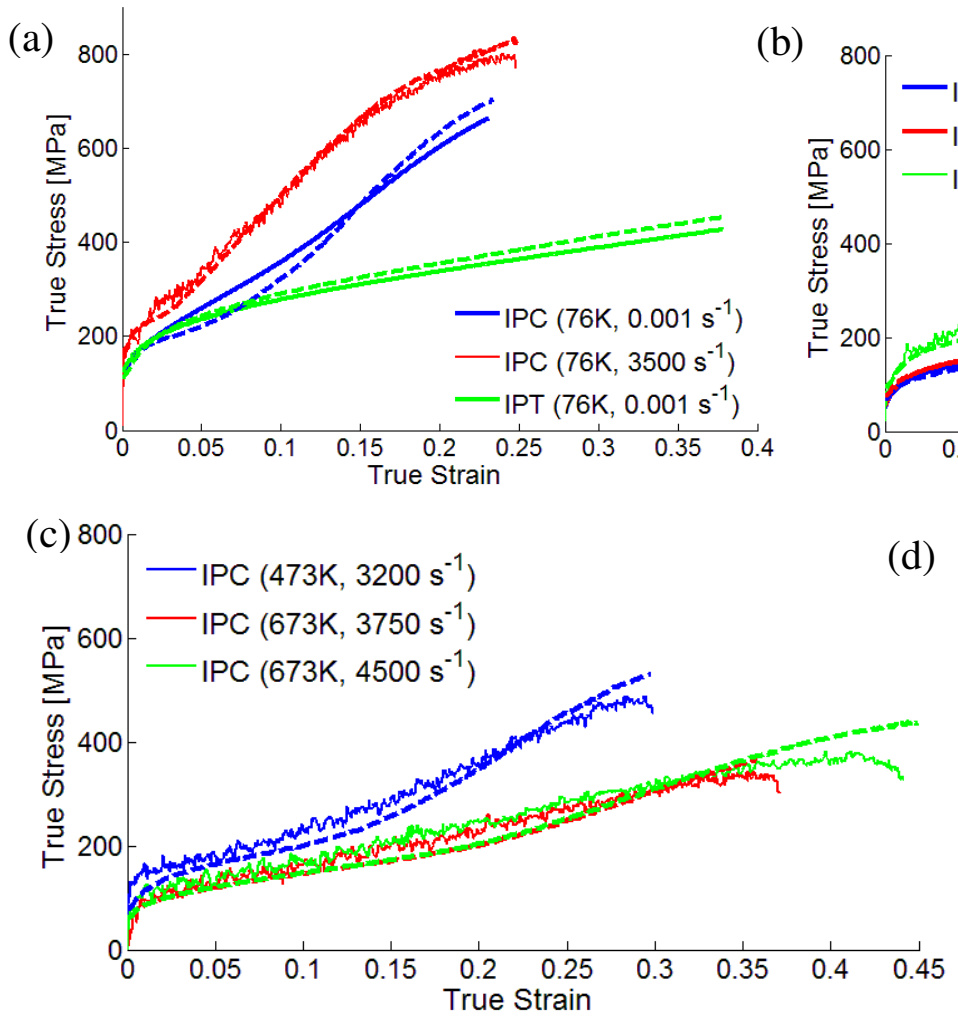

(e)

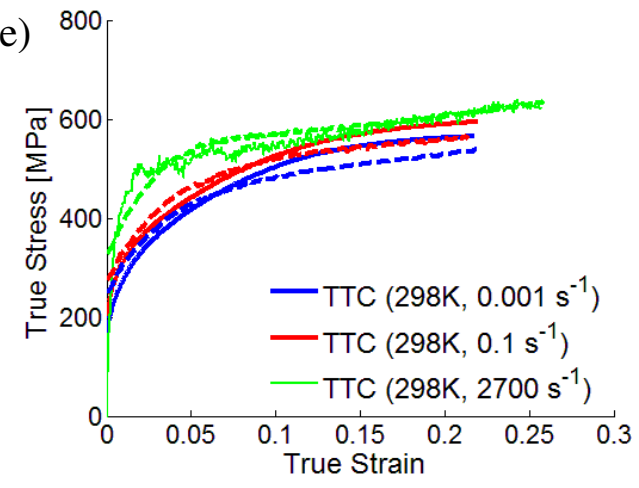

(b)

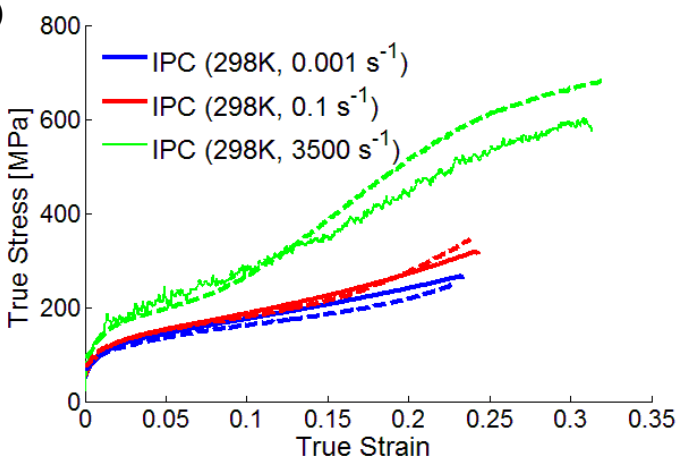

(d)

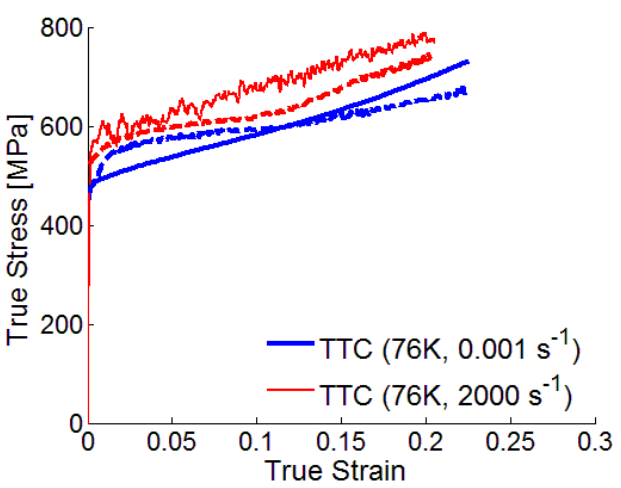

(f)

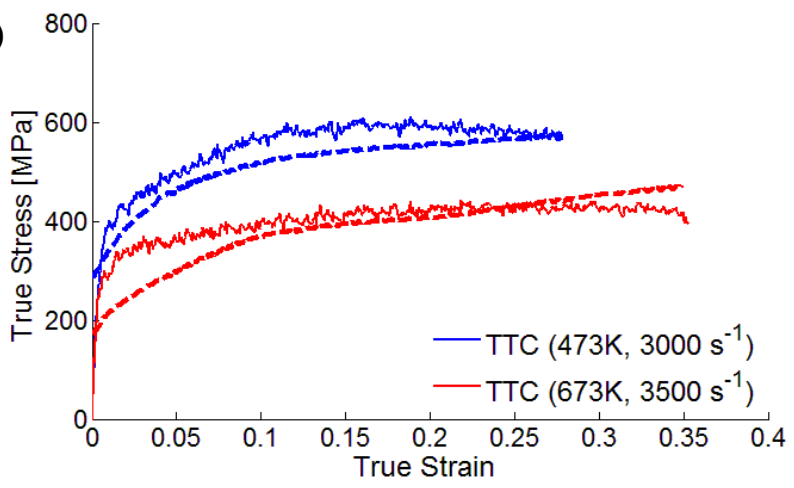

Figure 2 True stress-true strain responses of clock-rolled and annealed samples of $\alpha-\mathrm{Zr}$ as a function of temperature and strain rate. The loading direction is indicated: TTC is through thickness compression, IPC is in-plane compression, and IPT is in-plane tension. Measurements are solid lines and predictions are dashed lines. 

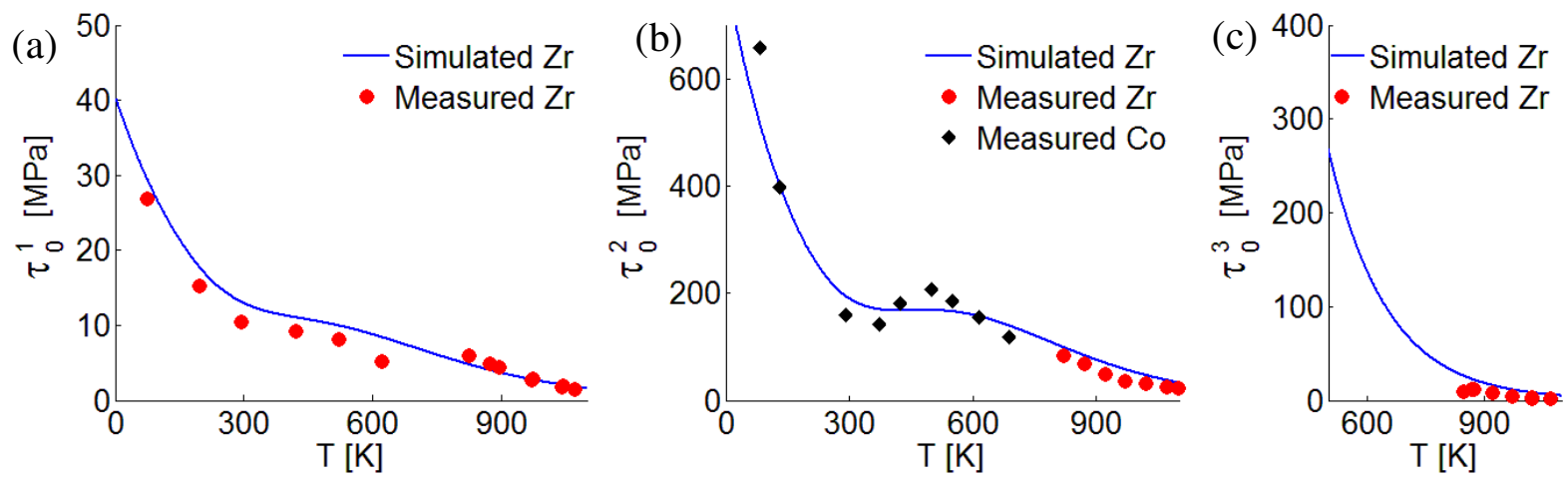

Figure 3 Variation of the initial slip resistance with temperature for (a) prismatic, (b) pyramidal $<\mathrm{c}+\mathrm{a}\rangle$, and (c) basal slip predicted by the model (lines) and data taken from the literature (symbols) [35-37, 78]. Data for Co [101] was divided by 2.5 to scale with Zr. 
(a) $45^{\circ}$ from IP1 $=$ IPC $\left(0.08,76 \mathrm{~K}, 0.001 \mathrm{~s}^{-1}\right)$

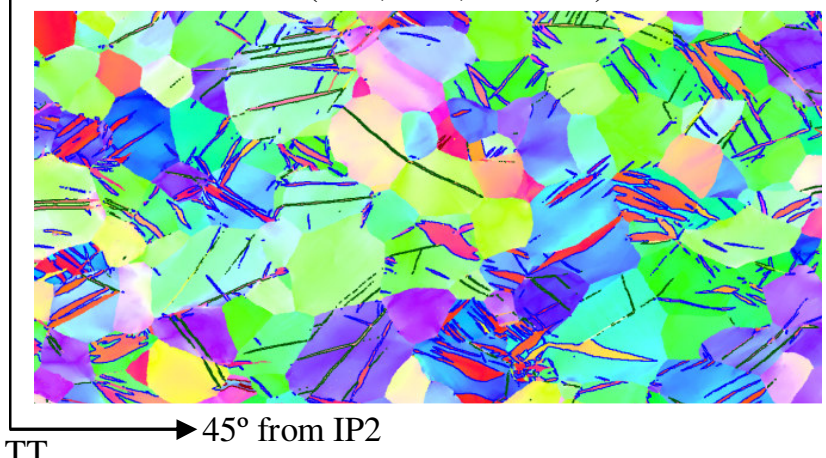

(b) $45^{\circ}$ from IP1= IPC $\left(0.065, \mathbf{2 9 8 K}, 0.001 \mathrm{~s}^{-1}\right)$

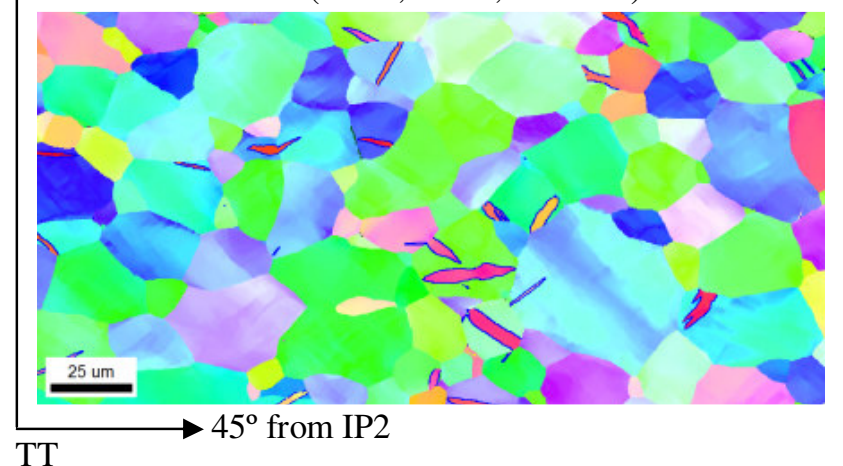

(d) $\operatorname{IP} 1=\operatorname{IPC}\left(0.44,673 \mathrm{~K}, 4500 \mathrm{~s}^{-1}\right)$

(c) $45^{\circ}$ from IP1 $1=\operatorname{IPC}\left(0.32, \mathbf{2 9 8 K}, 3500 \mathrm{~s}^{-1}\right)$

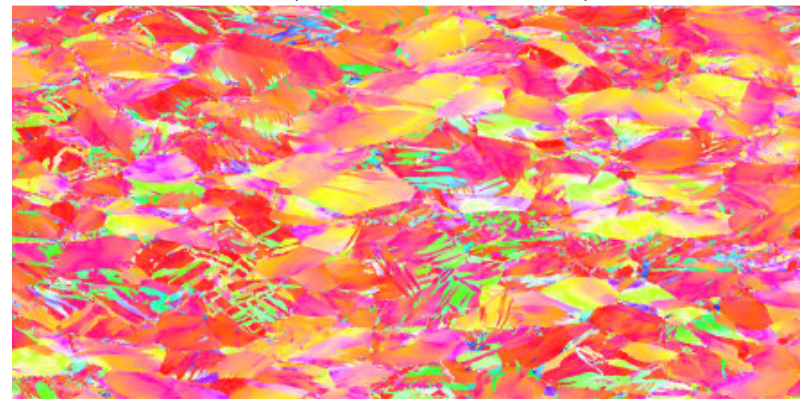

(e)
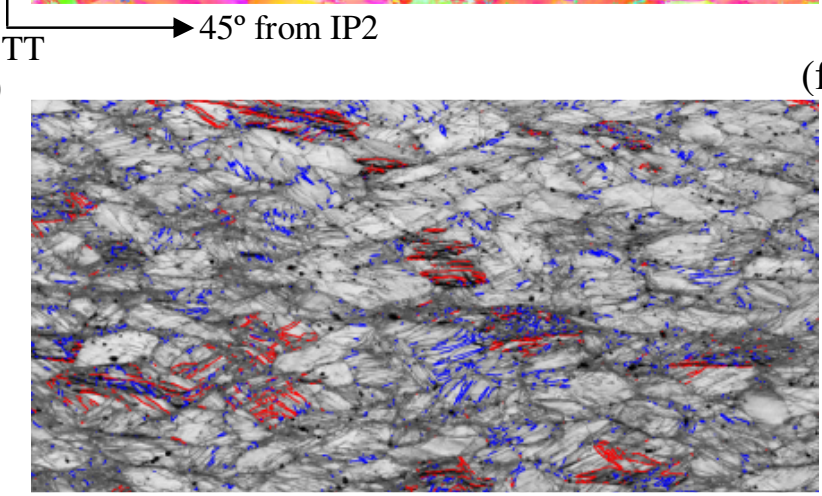

(f)
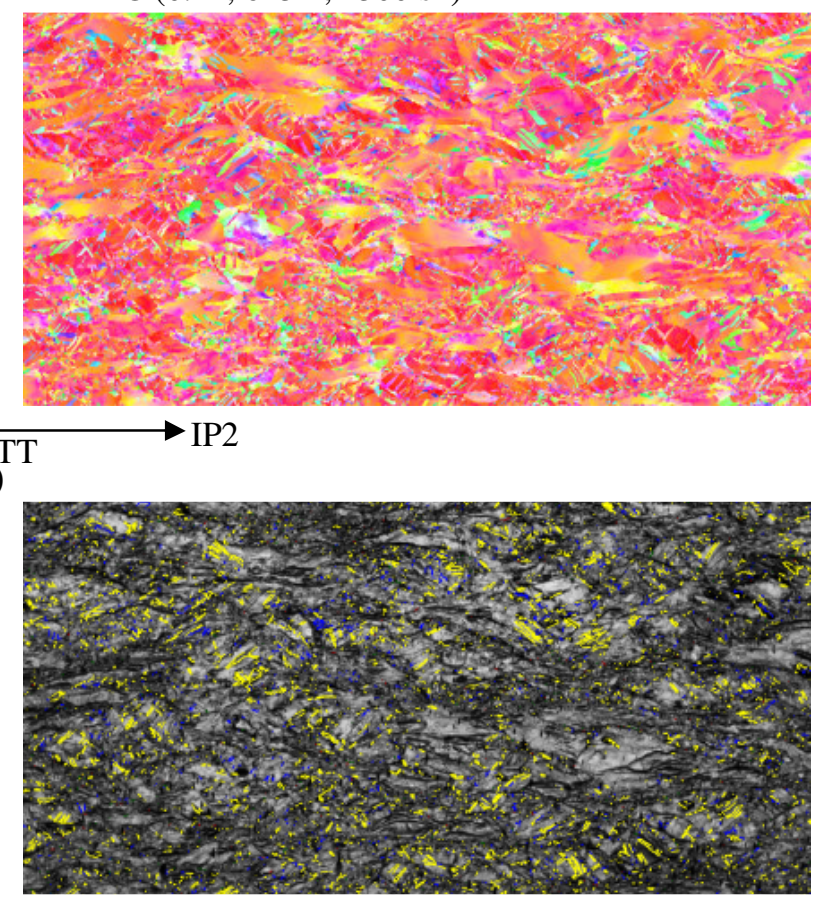

(g)

TT=TTC $\left(0.08,76 \mathbf{K}, 0.001 \mathrm{~s}^{-1}\right)$

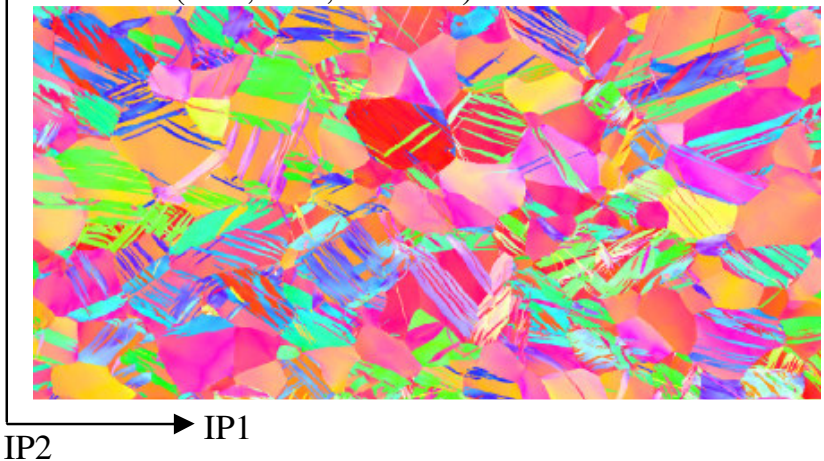

(h) $\mathrm{TT}=\mathrm{TTC}\left(0.34,673 \mathrm{~K}, 3500 \mathrm{~s}^{-1}\right)$

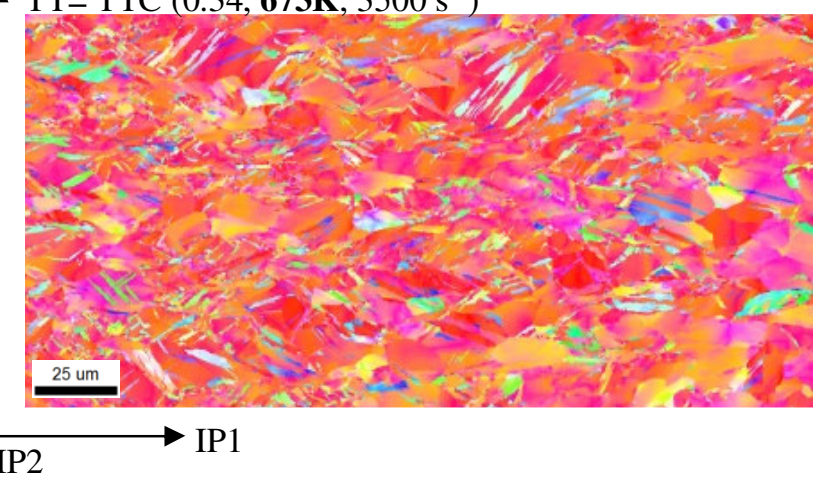


(i)

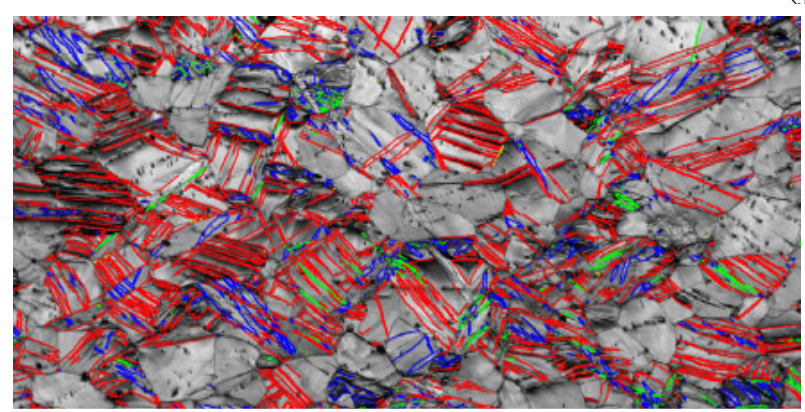

(i)
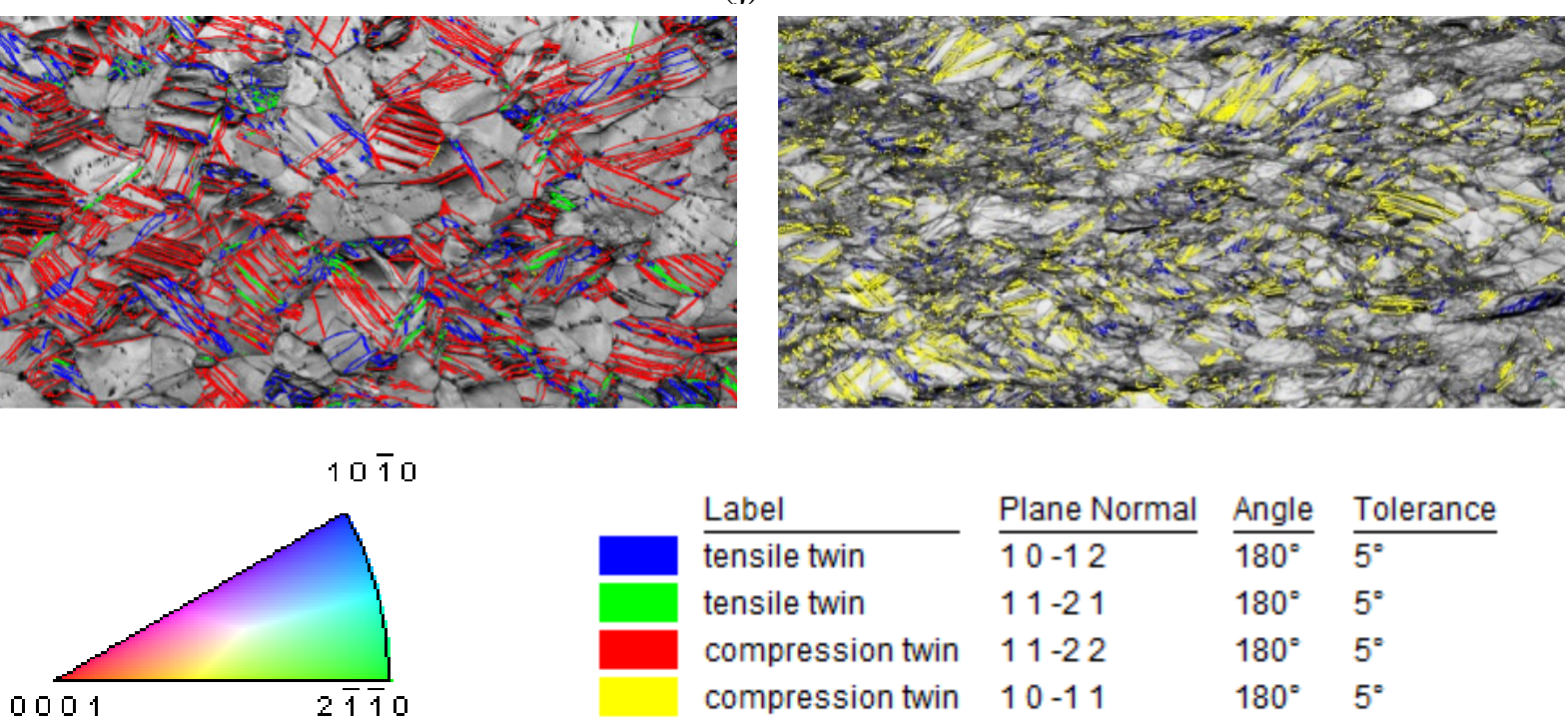

\begin{tabular}{llllll}
\cline { 1 - 1 } tensile twin & & Plane Normal & & Angle & Tolerance \\
tensile twin & $10-12$ & & $180^{\circ}$ & & $5^{\circ}$ \\
compression twin & $11-21$ & & $180^{\circ}$ & $5^{\circ}$ \\
compression twin & $10-11$ & & $180^{\circ}$ & $5^{\circ}$ \\
$180^{\circ}$ & $5^{\circ}$
\end{tabular}

Figure 4 Orientation maps showing the microstructures of the $\mathrm{Zr}$ samples deformed to various strain levels, at several temperatures and under several strain rates as indicated in the figure. The colors in the maps indicate the orientation of the compression axis with respect to the crystal reference frame according to the inverse pole figure (IPF) triangle. Twin boundaries for four twinning modes present in the maps are highlighted in the IPF maps for (a) and (b) and in the image quality maps (e), (f), (i) and (j) for corresponding IPF maps (c), (d), (g) and (h) with a different color as specified in the legend. The scale bar of $25 \mu \mathrm{m}$ on figure (b) is valid for all the maps. 

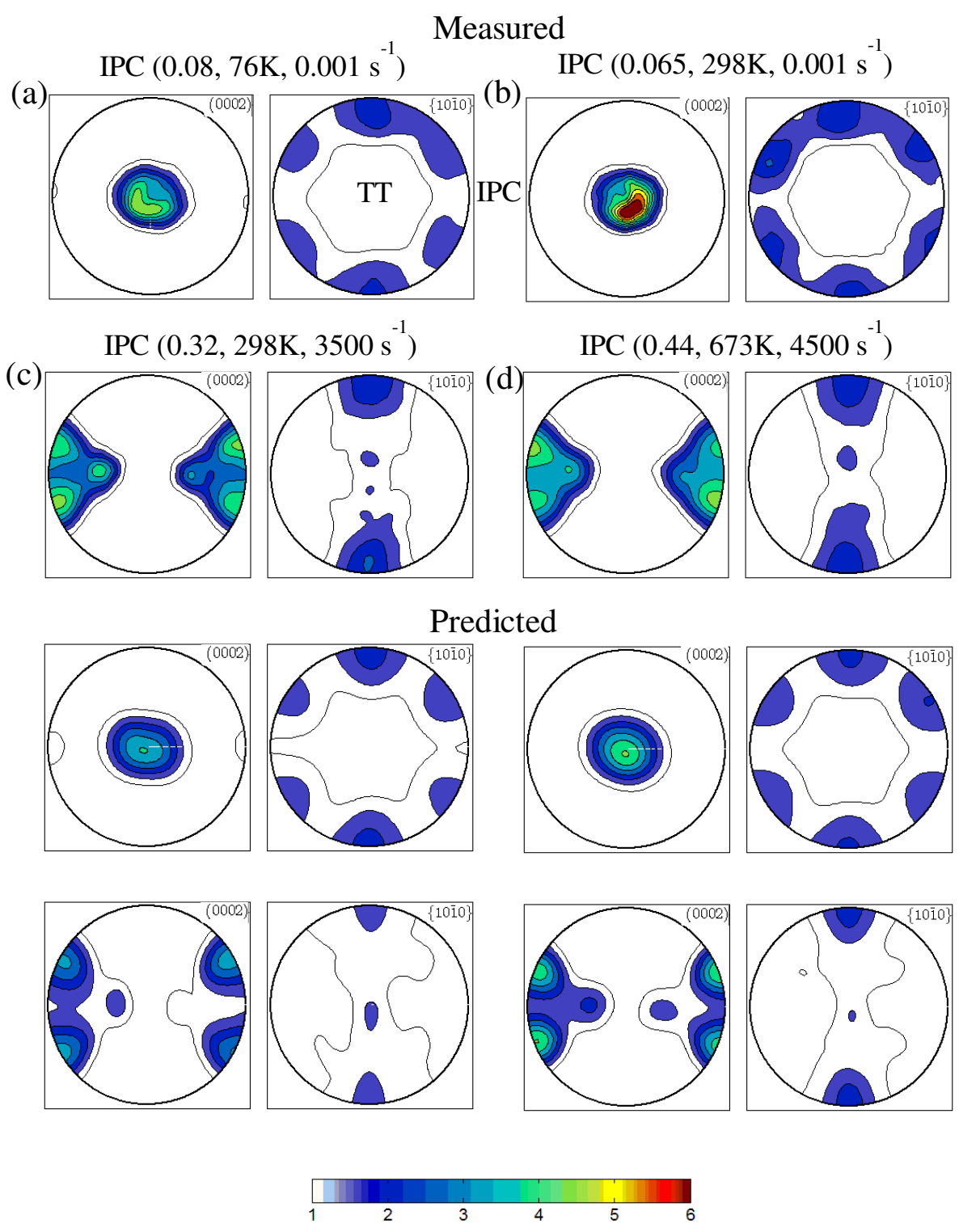

Figure 5 Basal and prismatic pole figures showing the measured and predicted texture evolution of $\alpha-\mathrm{Zr}$ deformed in in-plane compression (IPC) to the strain levels, temperatures and strain rates indicated in the figure. 

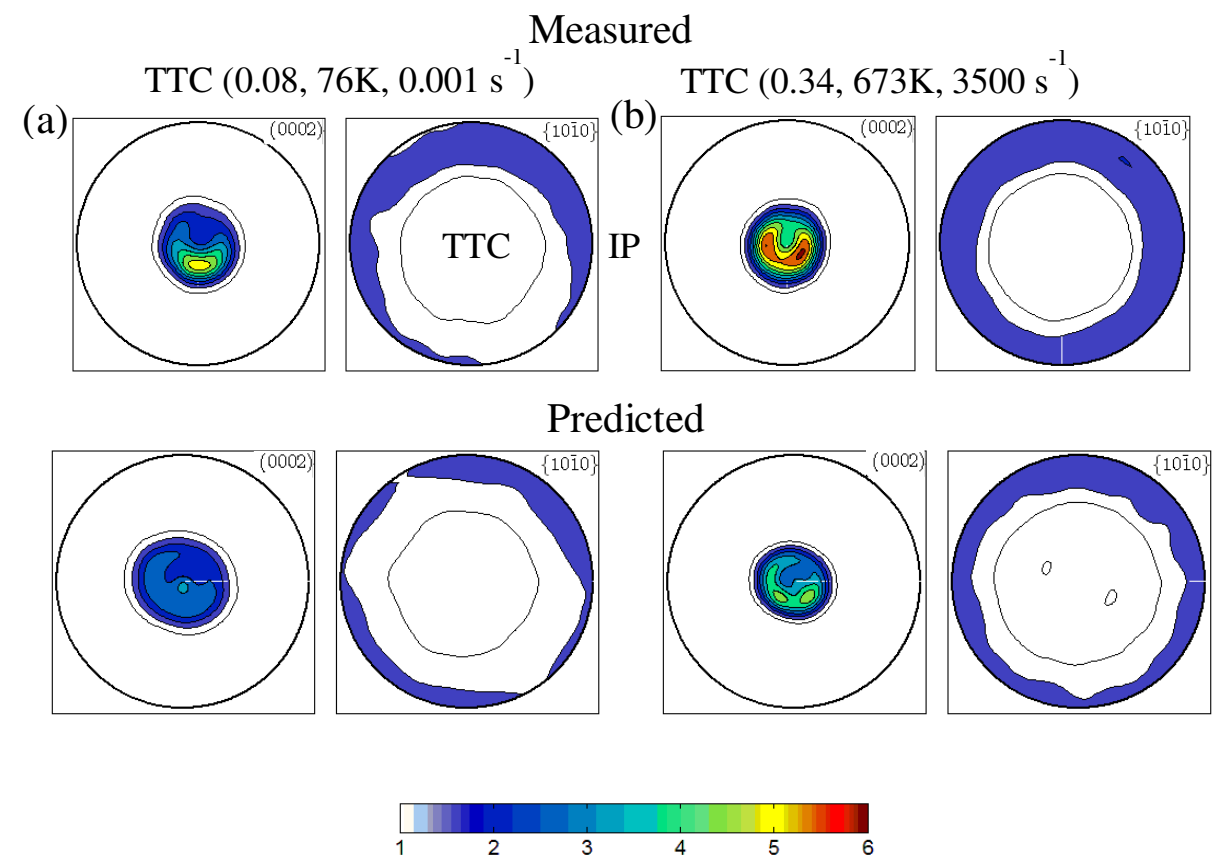

Figure 6 Basal and prismatic pole figures showing the measured and predicted texture evolution of $\alpha-\mathrm{Zr}$ deformed in through-thickness compression (TTC) to the strain levels, temperatures and strain rates as indicated in the figure.
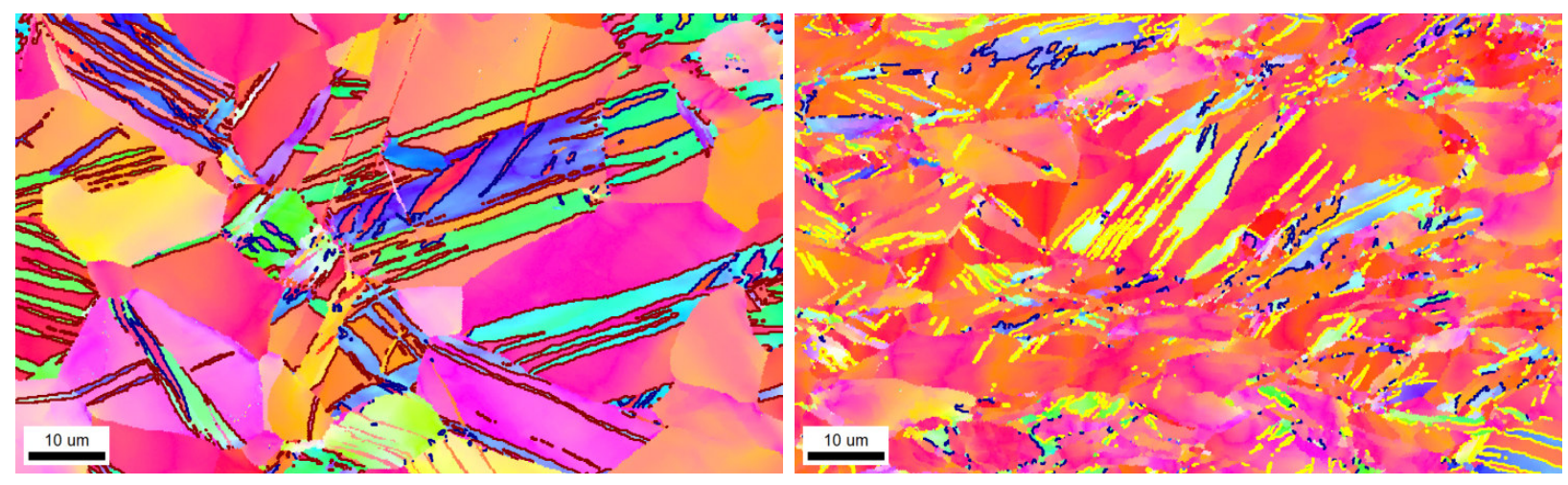

Figure 7 Selected regions of the orientation maps shown in Fig. 4g and h: (a) TTC at $76 \mathrm{~K}$ under $0.001 \mathrm{~s}^{-1}$ showing secondary $\{10 \overline{1} 2\}\langle 10 \overline{1} \overline{1}\rangle$ twins (blue boundaries) within the $\{11 \overline{2} 2\}\langle 11 \overline{2} \overline{3}\rangle$ primary twins (brown boundaries) and (b) TTC at $673 \mathrm{~K}$ under $3500 \mathrm{~s}^{-1}$ showing secondary $\{10 \overline{1} 2\}\langle 10 \overline{1} \overline{1}\rangle$ twins (blue boundaries) within the primary $\{10 \overline{1} 1\}\langle 10 \overline{1} \overline{2}\rangle$ twins. In (b), the regions (not boundaries) that are colored different than red are $\{10 \overline{1} 1\}\langle 10 \overline{1} \overline{2}\rangle$ twins. Twin boundaries are highlighted with the colors consistent with labeling scheme in the Fig. 4. 
(a) $\quad$ IPC $\left(298 \mathrm{~K}, 0.001 \mathrm{~s}^{-1}\right)$

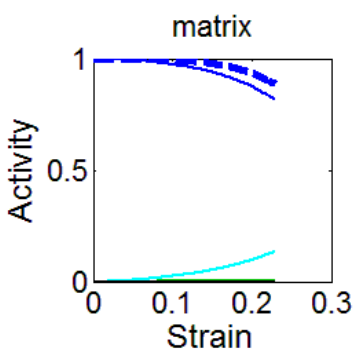

(c) IPC $\left(76 K, 3500 \mathrm{~s}^{-1}\right)$

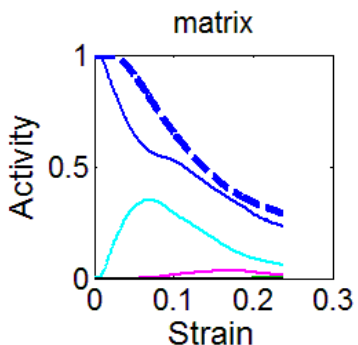

(e) $\quad$ IPC $\left(473 \mathrm{~K}, 3200 \mathrm{~s}^{-1}\right)$
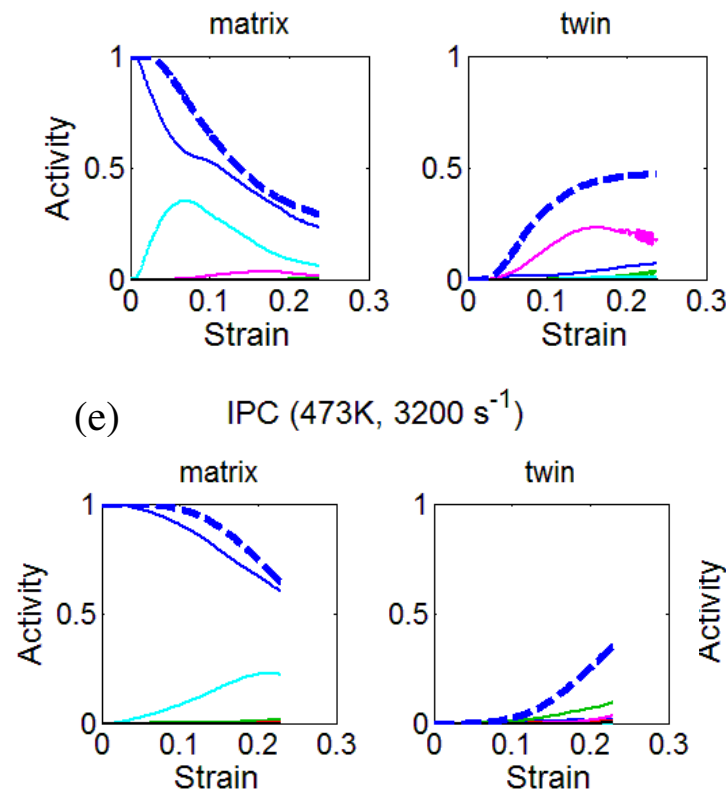

(d)
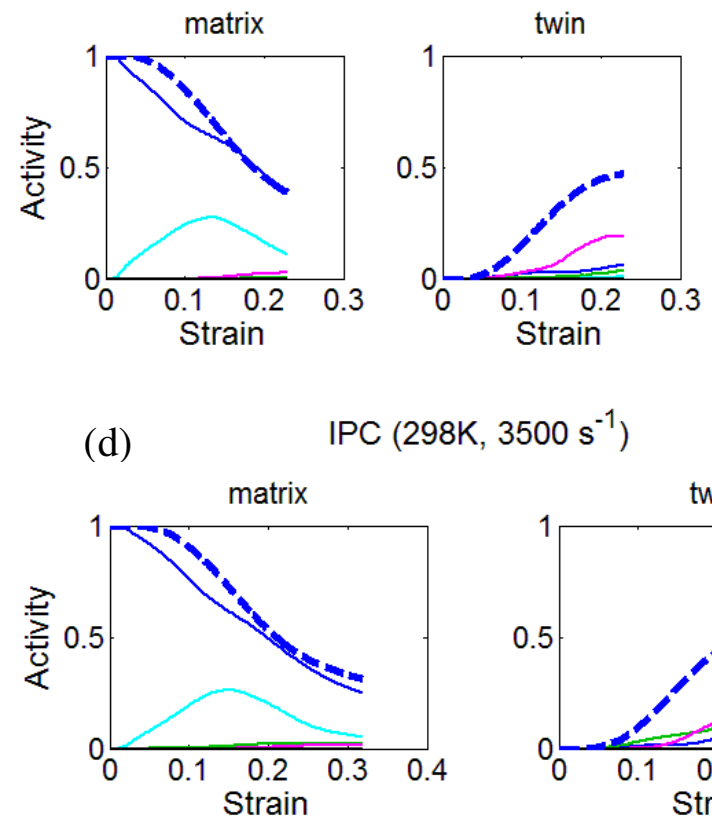

IPC $\left(298 \mathrm{~K}, 3500 \mathrm{~s}^{-1}\right)$

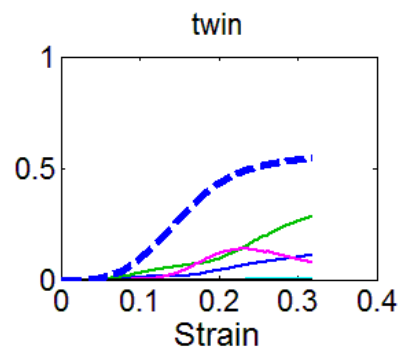

(f)

IPC (673K, $\left.4500 \mathrm{~s}^{-1}\right)$
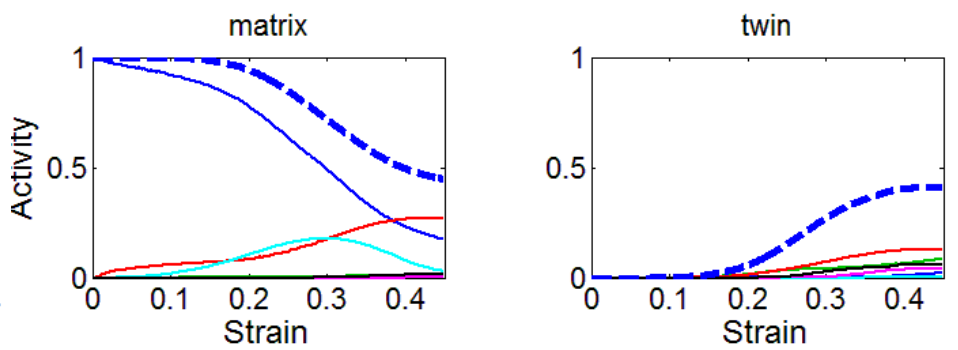

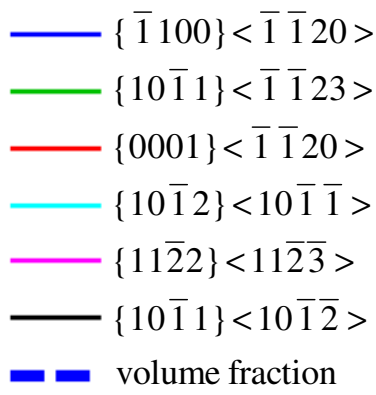

Figure 8 Predicted relative activities of each slip or twin mode contributing to plasticity in the parent grain and in the primary twin domains for the IP conditions indicated in the figure. Also plotted are the parent and the primary twinned material volume fractions. 

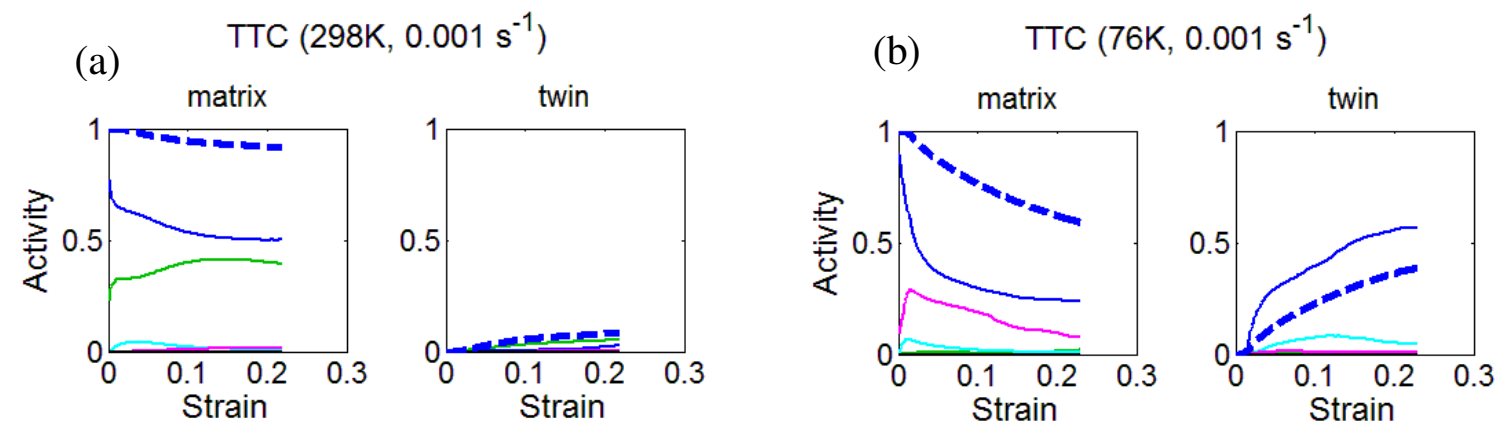

(c) $\quad \operatorname{TTC}\left(76 \mathrm{~K}, 2000 \mathrm{~s}^{-1}\right)$
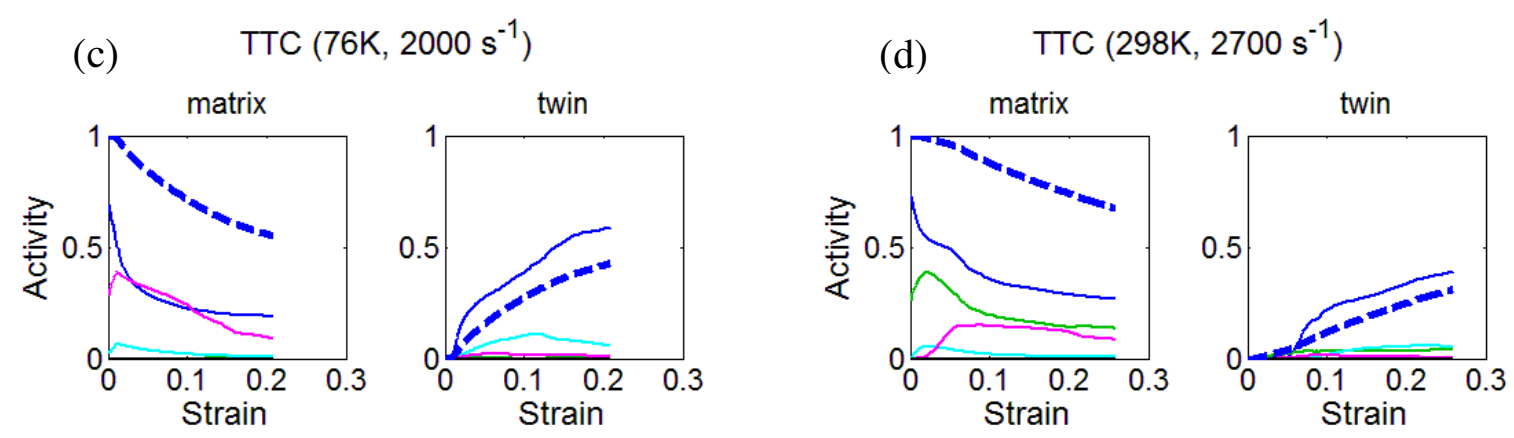

(e) TTC $\left(473 \mathrm{~K}, 3000 \mathrm{~s}^{-1}\right)$
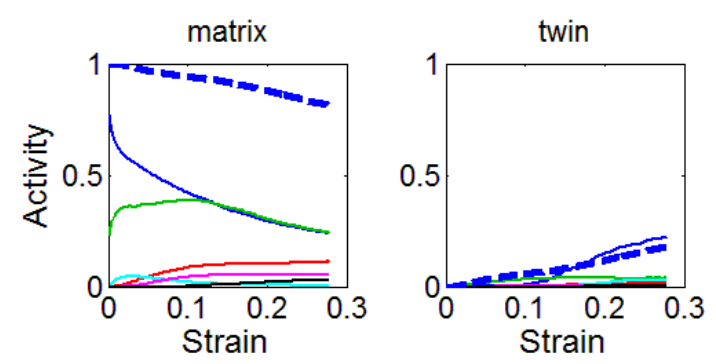

(f)

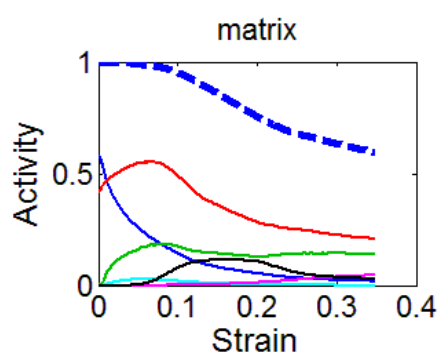

TTC $\left(673 \mathrm{~K}, 3500 \mathrm{~s}^{-1}\right)$

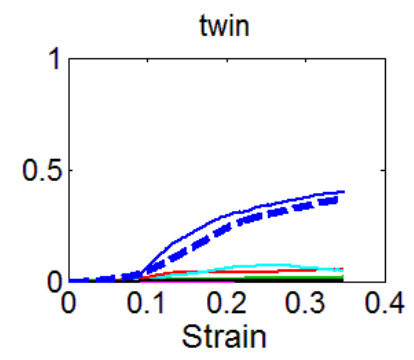

Figure 9 Predicted relative activities of each slip and twin mode contributing to plasticity in the parent grain and in the primary twin domains for the TT conditions indicated in the figure. Also plotted are the parent and the primary twinned material volume fractions. The same legend from Fig. 7 applies here. 


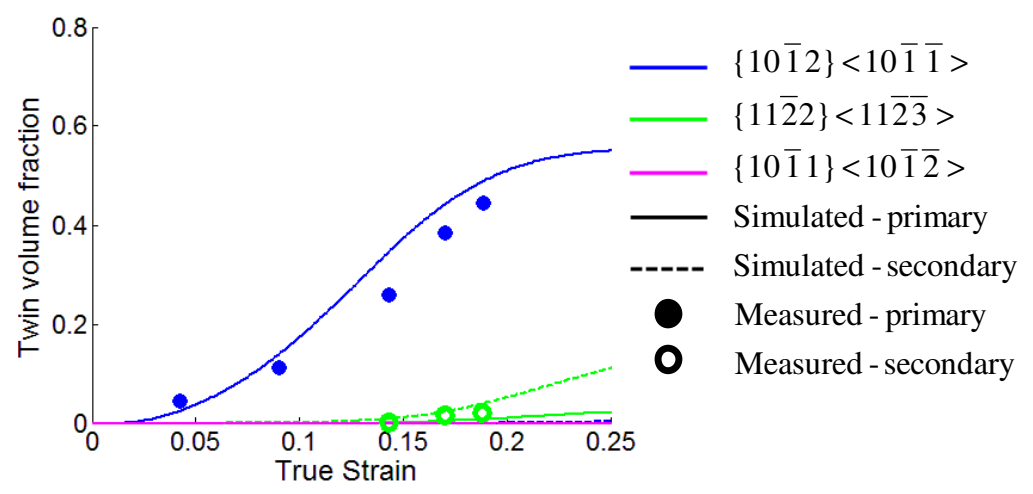

Figure 10 Evolution of the primary and secondary twin volume fraction during IPC and TTC at $76 \mathrm{~K}$ and under a strain rate of $0.001 \mathrm{~s}^{-1}$ predicted by VPSC (lines) and measured by EBSD (symbols) as a function of strain.

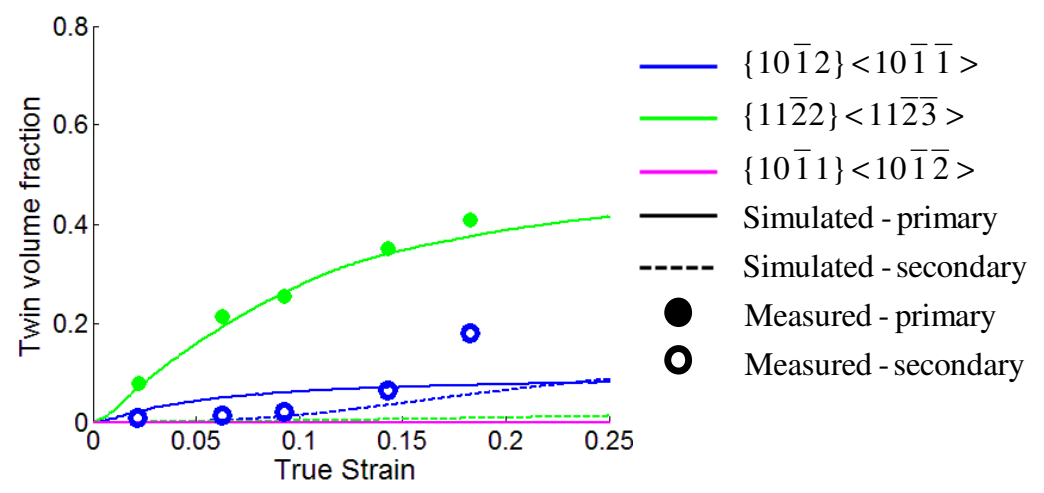

Figure 11 Evolution of the primary and secondary twin volume fraction during TTC at $76 \mathrm{~K}$ and under a strain rate of $0.001 \mathrm{~s}^{-1}$ predicted by VPSC (lines) and measured by EBSD (symbols) as a function of strain. 
(a)

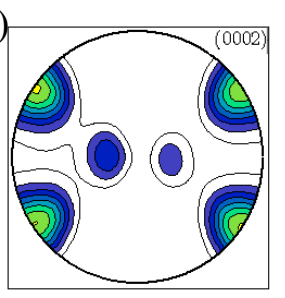

(b)

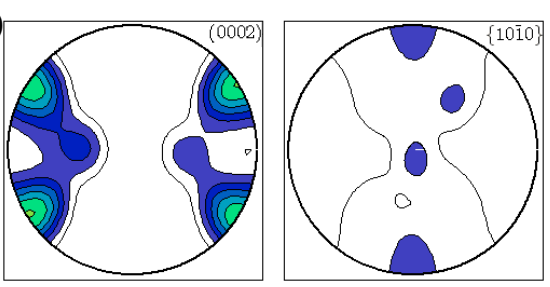

(c)
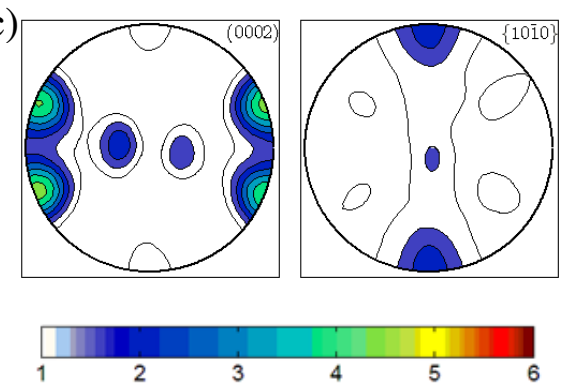
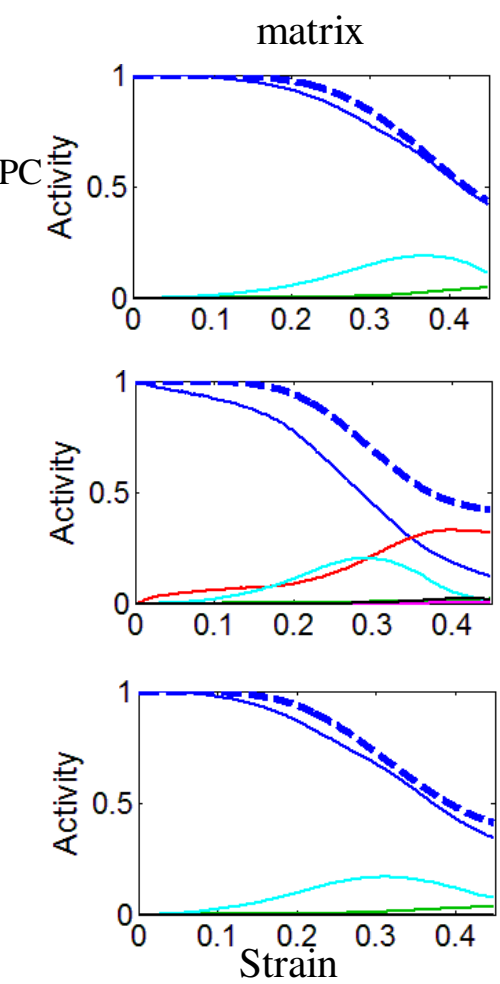

twin
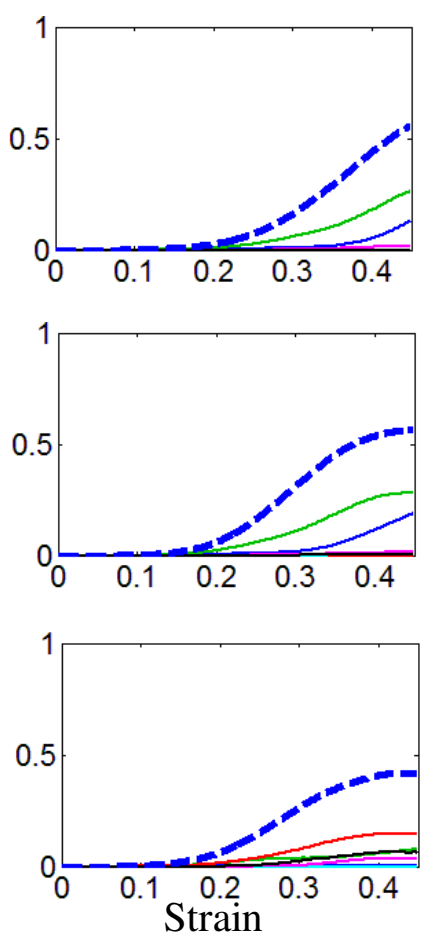

Figure 12 Effect of basal slip on texture evolution at $673 \mathrm{~K}$ (a) without basal slip (b) with primary basal slip in the parent grains (c) with secondary basal slip in the twinned regions. Basal slip both in parent and twinned regions were allowed in the results presented in Figs. 2, 5 and 8. 
Table 1: The matrix of tests: IPC - in-plane compression, IPT - in-plane tension, and TTC through-thickness compression at specified strain rate and temperature conditions that were carried out in this work. ${ }^{\dagger}$ indicates that multiple samples were deformed and tests interrupted at several strain levels for microstructural examination.

\begin{tabular}{|c|c|c|c|c|c|c|c|c|c|}
\hline \multicolumn{1}{c}{} & $0.001 \mathrm{~s}^{-1}$ & $0.1 \mathrm{~s}^{-1}$ & $2000 \mathrm{~s}^{-1}$ & $2700 \mathrm{~s}^{-1}$ & $3000 \mathrm{~s}^{-1}$ & $3200 \mathrm{~s}^{-1}$ & $3500 \mathrm{~s}^{-1}$ & $3750 \mathrm{~s}^{-1}$ & $4500 \mathrm{~s}^{-1}$ \\
\hline $76 \mathrm{~K}$ & $\begin{array}{c}\mathrm{IPC}^{\dagger}, \\
\mathrm{TTC}^{\dagger}, \\
\text { IPT }\end{array}$ & - & TTC & - & - & - & IPC & - & - \\
\hline $298 \mathrm{~K}$ & $\begin{array}{c}\text { IPC, } \\
\text { TTC }\end{array}$ & $\begin{array}{c}\text { IPC, } \\
\text { TTC }\end{array}$ & - & TTC & - & - & IPC & - & - \\
\hline $473 \mathrm{~K}$ & - & - & - & - & TTC & IPC & - & - & - \\
\hline $673 \mathrm{~K}$ & - & - & - & - & - & - & TTC & IPC & IPC \\
\hline
\end{tabular}


Table 2 Material parameters used in the model for the evolution of slip system resistance.

\begin{tabular}{|c|c|c|c|c|c|c|}
\hline \multirow{2}{*}{\multicolumn{2}{|c|}{$b^{\alpha}[m]$}} & \multicolumn{2}{|c|}{$\alpha=1\{\overline{1} 100\}\langle\overline{1} \overline{1} 20\rangle$} & \multicolumn{2}{|c|}{$\alpha=2\{10 \overline{1} 1\}\langle\overline{1} \overline{1} 23\rangle$} & \multirow{2}{*}{$\begin{array}{l}\alpha=3\{0001\}\langle\overline{1} \overline{1} 20\rangle \\
3.231 \times 10^{-10}\end{array}$} \\
\hline & & \multicolumn{2}{|l|}{$3.231 \times 10^{-10}$} & \multicolumn{2}{|c|}{$6.0771 \times 10^{-10}$} & \\
\hline \multicolumn{2}{|c|}{$\tau_{0}^{\alpha}[M P a]$} & Eq. 3 & & \multicolumn{2}{|l|}{ Eq. 3} & Eq.3 \\
\hline \multicolumn{2}{|c|}{$H P^{\alpha}$} & 100 & & 170 & & 100 \\
\hline \multicolumn{2}{|c|}{$k_{1}^{\alpha}\left[m^{-1}\right]$} & \multicolumn{2}{|l|}{$5.7 \times 10^{8}$} & \multicolumn{2}{|l|}{$3.0 \times 10^{9}$} & $5.7 \times 10^{9}$ \\
\hline \multicolumn{2}{|c|}{$g^{\alpha}$} & \multicolumn{2}{|l|}{0.002} & \multicolumn{2}{|l|}{0.005} & 0.002 \\
\hline \multicolumn{2}{|c|}{$D^{\alpha}[M P a]$} & \multicolumn{2}{|l|}{700} & \multicolumn{2}{|l|}{1400} & 700 \\
\hline \multicolumn{2}{|c|}{$q^{\alpha}$} & \multicolumn{2}{|c|}{$12 \log (1+T / 30)$} & \multicolumn{2}{|l|}{0.1} & $12 \log (1+T / 30)$ \\
\hline & \multicolumn{2}{|c|}{$\alpha=1\{\overline{1} 100\}\langle\overline{1} \overline{1} 20\rangle$} & \multicolumn{2}{|c|}{$\alpha=2\{10 \overline{1} 1\}\langle\overline{1} \overline{1} 23\rangle$} & \multicolumn{2}{|c|}{$\alpha=3\{0001\}\langle\overline{1} \overline{1} 20\rangle$} \\
\hline $\mathrm{A}$ & \multicolumn{2}{|c|}{40.5} & \multicolumn{2}{|c|}{800} & \multicolumn{2}{|c|}{7500} \\
\hline B & \multicolumn{2}{|l|}{0.04} & \multicolumn{2}{|l|}{0.02} & \multicolumn{2}{|l|}{ N/A } \\
\hline $\mathrm{C}$ & \multicolumn{2}{|l|}{100} & \multicolumn{2}{|l|}{190} & \multicolumn{2}{|l|}{150} \\
\hline D & \multicolumn{2}{|l|}{235} & \multicolumn{2}{|l|}{36000} & \multicolumn{2}{|l|}{ N/A } \\
\hline$\sigma$ & \multicolumn{2}{|l|}{7.2} & 7.45 & & N/A & \\
\hline$v$ & 0.5 & & 0.5 & & N/A & \\
\hline
\end{tabular}

Table 3 Material parameters used in the model for the evolution of twin resistance.

\begin{tabular}{llll} 
& $\beta=1\{10 \overline{1} 2\}\langle 10 \overline{1} \overline{1}\rangle$ & $\beta=2\{11 \overline{2} 2\}\langle 11 \overline{2} \overline{3}\rangle$ & $\beta=3\{10 \overline{1} 1\}\langle 10 \overline{1} \overline{2}\rangle$ \\
\hline$b^{\beta}[\mathrm{m}]$ & $6.33 \times 10^{-11}$ & $9.24 \times 10^{-11}$ & $2.56 \times 10^{-11}$ \\
$S$ & 0.167 & 0.225 & 0.104 \\
\hline$\tau_{\text {crit }}^{\beta}[\mathrm{MPa}]$ & 140 & 350 & 750 \\
$\tau_{\text {prop }}^{\beta}[\mathrm{MPa}]$ & 10 & 350 & 50 \\
$H P^{\beta}$ & 135 & 100 & 100 \\
$C^{\beta}$ & N/A & $1300-100 \log (\dot{\varepsilon})$ & N/A \\
$C^{2 \beta}$ & 100 & 10 & N/A \\
$C^{3 \beta}$ & N/A & $1300-100 \log (\dot{\varepsilon})$ & N/A
\end{tabular}

